\title{
Spatially Modulated Electronic Nematicity in the Three-Band Model of Cuprate Superconductors
}

\author{
S. Bulut, ${ }^{1,2}$ W. A. Atkinson, , , 因 and A. P. Kampf ${ }^{3}$ \\ ${ }^{1}$ Department of Physics and Astronomy, Trent University, Peterborough Ontario, Canada, K9J $7 B 8$ \\ ${ }^{2}$ Department of Physics, Queen's University, Kingston Ontario, Canada, KrL $3 N 6$ \\ ${ }^{3}$ Theoretical Physics III, Center for Electronic Correlations and Magnetism, \\ Institute of Physics, University of Augsburg, 86135 Augsburg, Germany
}

(Dated: April 30, 2018)

\begin{abstract}
Charge order in cuprate superconductors is a possible source of anomalous electronic properties in the underdoped regime. Intra-unit cell charge ordering tendencies point to electronic nematic order involving oxygen orbitals. In this context we investigate charge instabilities in the Emery model and calculate the charge susceptibility within diagrammatic perturbation theory. In this approach, the onset of charge order is signalled by a divergence of the susceptibility. Our calculations reveal three different kinds of order: a commensurate $(q=0)$ nematic order, and two incommensurate nematic phases with modulation wavevectors that are either axial or oriented along the Brillouin zone diagonal. We examine the nematic phase diagram as a function of the filling, the interaction parameters, and the band structure. We also present results for the excitation spectrum near the nematic instability, and show that a soft nematic mode emerges from the particle-hole continuum at the transition. The Fermi surface reconstructions that accompany the modulated nematic phases are discussed with respect to their relevance for magneto-oscillation and photoemission measurements. The modulated nematic phases that emerge from the three-band Emery model are compared to those found previously in one-band models.
\end{abstract}

\section{INTRODUCTION}

Cuprate superconductors are susceptible to spin and charge density ordered phases that compete with superconductivity. This is well established in Labased cuprates, $1-3$ where (quasi) static spin/charge stripes are widely observed, even in coexistence with superconductivity ${ }^{4}, 5$. However, their presence in other cuprate families is generally unconfirmed. Because density waves are one of the proposed origins for the pseudogap in the underdoped regime, it is necessary to establish whether charge and spin order are universal amongst the cuprates. The recent discovery of charge order (without spin order) in underdoped $\mathrm{YBa}_{2} \mathrm{Cu}_{3} \mathrm{O}_{6+\mathrm{x}}(\mathrm{YBCO})$ is an important step in this direction. In this work, we describe novel incommensurate charge-ordered phases that arise in the three-band Emery model for cuprates, and discuss the extent to which these are consistent with the charge order detected in YBCO.

Early evidence for broken symmetry phases in YBCO came from magneto-oscillation experiments, which identified a reconstructed Fermi surface $e^{6,7}$ with an electronlike Fermi surface pocket $\underline{8}$ that emerges when strong magnetic fields are applied. These experiments were theoretically discussed from the perspective of density waves $\frac{9}{}$ Nernst effect measurements found a uniaxial symmetry breaking, ${ }^{10}$ consistent with a charge-density wave (CDW). Subsequent nuclear magnetic resonance (NMR) experiments further suggested a commensurate CDW with a period of four unit cells, with no evidence for any spin ordering ${ }^{11}$. In this work, the authors made a clear distinction between ortho-II YBCO (with hole doping $p \sim 0.108-0.12$ ) where only charge order is seen, and lower dopings near the superconductor-insulator transi- tion where charge and spin order are both seen. ${ }^{12-14}$

More recently, $\mathrm{x}$-ray scattering ${ }^{15-17}$ experiments have identified a CDW phase in the same doping and magnetic field range in which Fermi surface pockets are detected. The charge pattern is aligned with the crystalline axes ${ }^{15,16}$ and is incommensurate, with a weakly doping dependent period of $\sim 3.2$ lattice constants. Whether this CDW is uniaxial10,11 or biaxial18,19 has not been resolved, and may depend on doping 17 Regardless of the details, X-ray and NMR experiments established that the incommensurate CDW competes with the superconductivity, $\stackrel{16,20}{2}$ implying that both phases operate on similar energy scales.

The x-ray results suggest that this charge-ordered state is distinct from the stripe phase in La-based cuprates; $;, 17,19$ however, its relation to apparent chargeordered phases in Bi-based cuprates is still not clear. Photoemission experiments on underdoped Bi-cuprates have found spectral features ${ }^{21-24}$ that are consistent with competing non-superconducting phases. Scanning tunneling microscopy (STM) experiments ${ }^{25}-31$ further pointed to intra-unit cell charge order. The simplest candidates for such order are uniaxial "nematic", or biaxial "checkerboard" phases involving a spontaneous transfer of charge between oxygen $p_{x}$ and $p_{y}$ orbitals within the $\mathrm{CuO}_{2}$ unit cell.

In the present work, we report on possible chargeordered phases within the three-band Emery model,,$^{32}$ which includes copper $d$ and oxygen $p$ orbitals. We have calculated the charge susceptibility $\chi_{\alpha \beta}(\mathbf{q}, \omega)(\alpha$ and $\beta$ are orbital indices) diagrammatically. ${ }^{33,34}$ The leading instabilities of the model are obtained from divergences of the static susceptibility $\chi_{\alpha \beta}(\mathbf{q})$. We find that, through much of the phase diagram, the leading instability is to 
an incommensurate (finite- $q$ ) charge modulation involving primarily the oxygen orbitals. When the ordering wavevector $\mathbf{q}^{*}$ tends to zero, this phase evolves continuously into the commensurate nematic phase proposed by Fischer and $\mathrm{Kim}^{35}$ to explain the STM results for Bicuprates.

The possibility of finite- $q$ "modulated nematic order" (MNO) was raised previously in one-band models $\underline{36}-\underline{42}$ In this context, "nematic" refers to an anisotropic renormalization of the bond-centered kinetic energy that disrupts the tetragonal symmetry of the lattice. This anisotropy can be viewed as the result of integrating out inequivalent oxygen orbitals in a three-band model,$\underline{40}$ and in this light the one-band bond-centered nematicity may be related to the nematic phase reported here, which is characterized by a spontaneous transfer of charge between $\mathrm{O} p_{x}$ and $\mathrm{O} p_{y}$ orbitals. Note that we make a distinction between MNO, for which the charge transfer is predominantly intra-unit cell, and conventional CDW order, for which the charge transfer is inter-unit cell.

Despite the differences between one- and three-band models, there are some surprising similarities in the structure of the modulations. Within the "hotspot" model $l^{36,40}$ the modulation wavevector $\mathbf{q}^{*}$ lies along the Brillouin zone diagonal. Holder and Metzner $\underline{39}$ considered a more general interaction and found that $\mathbf{q}^{*}$ may be either diagonal or axial (bond-aligned), depending on the Fermi-surface shape. Here, we find that the doping dependence of $\mathbf{q}^{*}$ is qualitatively the same as that found by Holder and Metzner, even though the mechanism which drives the instability is different.

Earlier, a different charge instability, involving charge transfer between $\mathrm{Cu}$ and $\mathrm{O}$ was found in the three-band Emery model when the energies of $\mathrm{O} p_{x}, \mathrm{O} p_{y}$, and $\mathrm{Cu} d$ orbitals are close to degenerate $33,34,43$ This charge transfer instability does not occur for the more realistic range of parameters studied below. In the strong-coupling limit of the Emery model it was instead argued that the interaction $V_{p p}$ between neighboring oxygen sites confines the motion of doped holes to one-dimensional channels which thereby suggests a possible source for nematicity $\underline{44}$. A continuum theory for the quantum phase transition to the nematic state was subsequently developed $\underline{45}$.

We describe the model and the diagrammatic calculations in detail in Sec. II results are presented in Sec. III From the static nematic susceptibility, we obtain phase diagrams as functions of temperature, doping, and interaction strengths. We show that, for realistic model parameters, there exists a wide doping range over which incommensurate nematic order is preferred over commensurate order. The results for the dynamical susceptibility show that the nematic transition is marked by a soft mode that emerges from the particle-hole continuum near the nematic instability. We also describe the expected Fermi-surface reconstruction in the nematic phase. In Sec.IV we compare one- and three-band models showing why they generate similar phase diagrams despite significant differences in the models. Finally we discuss to

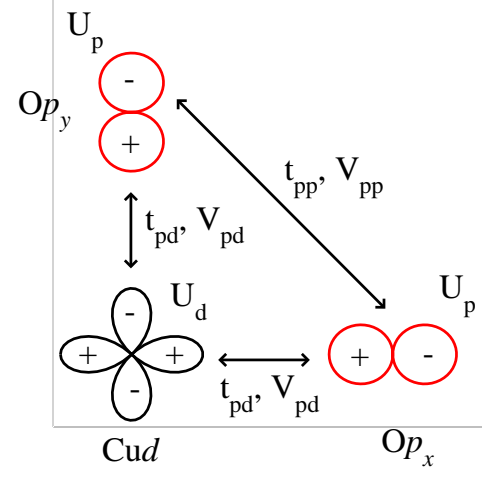

FIG. 1: (Color online) Unit cell for the three-band Emery model. The model includes one copper $d_{x^{2}-y^{2}}$ orbital along with two oxygen orbitals, denoted by $\mathrm{O} p_{x}$ and $\mathrm{O} p_{y}$. Hopping matrix elements $\left(t_{p d}\right.$ and $\left.t_{p p}\right)$ and Coulomb interaction strengths $\left(U_{d}, U_{p}, V_{p d}, V_{p p}\right)$ are indicated. Throughout we fix $t_{p d}=1, U_{d}=9, U_{p}=3$, and $V_{p d}=1$.

what extent our results for modulated nematic order are compatible with the existing experimental evidence for charge ordering.

\section{MODEL AND CALCULATIONS}

In this section we describe briefly the three-band Emery mode ${ }^{32}$ and outline the calculation of the charge susceptibility matrix $\chi_{\alpha \beta}(\mathbf{q}, \omega)$.

\section{A. Hamiltonian}

The unit cell of a single $\mathrm{CuO}_{2}$ plane is illustrated in Fig. 1. It consists of three orbitals, one copper $d_{x^{2}-y^{2}}$ orbital and two oxygen orbitals labeled $\mathrm{O} p_{x}$ and $\mathrm{O} p_{y}$ that form $\sigma$-bonds with the $\mathrm{Cu} d$ orbital. The noninteracting tight-binding Hamiltonian in momentum space is given by

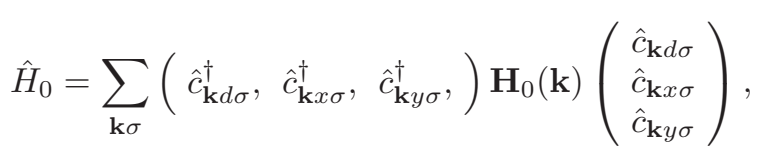

where $\hat{c}_{\mathbf{k} \alpha \sigma}$ is the annihilation operator for an electron with wavevector $\mathbf{k}$, spin $\sigma$, and orbital index $\alpha$; $\alpha=$ $d, x, y$ indicates $\mathrm{Cu} d, \mathrm{O} p_{x}, \mathrm{O} p_{y}$ orbitals, respectively. In the matrix elements of

$$
\mathbf{H}_{0}(\mathbf{k})=\left(\begin{array}{ccc}
\epsilon_{d} & 2 t_{p d} s_{x} & -2 t_{p d} s_{y} \\
2 t_{p d} s_{x} & \epsilon_{x} & -4 t_{p p} s_{x} s_{y} \\
-2 t_{p d} s_{y} & -4 t_{p p} s_{x} s_{y} & \epsilon_{y}
\end{array}\right),
$$

with $s_{x}=\sin \left(k_{x} / 2\right), s_{y}=\sin \left(k_{y} / 2\right), t_{p d}$ and $t_{p p}$ are the nearest neighbor $p$ - $d$ and $p-p$ hopping amplitudes (see Fig. (1). $\epsilon_{d}$ denotes the Cud orbital energy and $\epsilon_{x}$ and 
$\epsilon_{y}$ the corresponding energies of the $\mathrm{O} p_{x}$ and $\mathrm{O} p_{y}$ orbitals. In calculating $\mathbf{H}_{0}$, the signs of the hopping matrix elements are determined by the phases of the nearest atomic wavefunction lobes (indicated by "+" and "-" in Figure 11) for a given bond. For the $H_{0,23}$ and $H_{0,32}$ matrix elements, we introduced an extra minus sign as that enables us to obtain a realistic Fermi surface for a non-zero $t_{p p}$ value. Such a sign change can arise from indirect hopping through the Cu4s orbital $\underline{\underline{46}}$ Unless otherwise specified, we take $\epsilon_{x}=\epsilon_{y} \equiv \epsilon_{p}$, choose $t_{p d}=1$ as the unit of energy, and adopt the common estimate for the charge transfer energy $\Delta_{C T}=\epsilon_{d}-\epsilon_{p}=2.5 \underline{47}$. To understand the role of the Fermi-surface shape, we consider two cases: $t_{p p}=0$ and $t_{p p}=0.5$. The former gives a highly nested Fermi surface, while the latter is consistent with band-structure calculations for the cuprates $\underline{\underline{48}}$

The energy eigenvalues and eigenvectors follow from the diagonalization of $\mathbf{H}_{0}(\mathbf{k})$,

$$
\mathbf{S}^{\dagger}(\mathbf{k}) \mathbf{H}_{0}(\mathbf{k}) \mathbf{S}(\mathbf{k})=\mathbf{\Lambda}(\mathbf{k}),
$$

where $\Lambda_{i j}(\mathbf{k})=\delta_{i j} E_{\mathbf{k}}^{i}$ is the diagonal eigenvalue matrix containing the band energies $E_{\mathbf{k}}^{i}$, and $\mathbf{S}(\mathbf{k})$ is a $3 \times 3$ matrix of eigenvectors.

The interaction term in the Hamiltonian includes intraand inter-orbital Coulomb interactions. We consider intra-orbital interactions $U_{d}$ and $U_{p}$ at the $\mathrm{Cu} d$ and $\mathrm{O} p$ orbitals and inter-orbital interactions $V_{p d}$ and $V_{p p}$ between nearest neighbor $p$ - $d$ and $p$ - $p$ orbitals, respectively, as illustrated in Fig. 1]

The interaction term is

$$
\hat{V}=\frac{1}{2 N} \sum_{\alpha \beta} \sum_{\mathbf{q}} V_{\alpha \beta}(\mathbf{q}) \hat{n}_{\alpha}(\mathbf{q}) \hat{n}_{\beta}(-\mathbf{q})
$$

where $\hat{n}_{\alpha}(\mathbf{q})=\sum_{\sigma \mathbf{k}} \hat{c}_{\mathbf{k} \alpha \sigma}^{\dagger} \hat{c}_{\mathbf{k}+\mathbf{q} \alpha \sigma}$ and

$$
V_{\alpha \beta}(\mathbf{q})=\sum_{\mathbf{R}} e^{-i \mathbf{q} \cdot\left(\mathbf{R}+\mathbf{r}_{\beta}-\mathbf{r}_{\alpha}\right)} V_{\alpha \beta}(\mathbf{R}) .
$$

$\mathbf{R}$ denotes the lattice vectors and $\mathbf{r}_{\alpha}, \mathbf{r}_{\beta}$ the positions of orbitals $\alpha, \beta$ within the unit cell. Explicitly,

$$
\begin{aligned}
V_{\alpha \beta}(\mathbf{q}) & =\delta_{\alpha, d} \delta_{\beta, d} U_{d} \\
& +\left(\delta_{\alpha, x} \delta_{\beta, x}+\delta_{\alpha, y} \delta_{\beta, y}\right) U_{p} \\
& +\left(\delta_{\alpha, x} \delta_{\beta, d}+\delta_{\alpha, d} \delta_{\beta, x}\right) 2 V_{p d} \cos \left(q_{x} / 2\right) \\
& +\left(\delta_{\alpha, y} \delta_{\beta, d}+\delta_{\alpha, d} \delta_{\beta, y}\right) 2 V_{p d} \cos \left(q_{y} / 2\right) \\
& +\left(\delta_{\alpha, y} \delta_{\beta, x}+\delta_{\alpha, x} \delta_{\beta, y}\right) 4 V_{p p} \cos \left(q_{x} / 2\right) \cos \left(q_{y} / 2\right) .
\end{aligned}
$$

Throughout this paper, we set $U_{d}=9, U_{p}=3$, and $V_{p d}=1$ (in units of $\left.t_{p d}\right) \underline{\underline{48}}$

\section{B. Charge Susceptibility}

In order to detect tendencies for nematic instabilities, we consider the order parameter

$$
O_{N}(\mathbf{q})=n_{x}(\mathbf{q})-n_{y}(\mathbf{q}),
$$

(a)

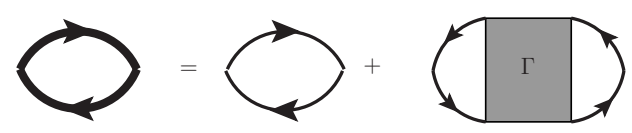

$(b)$

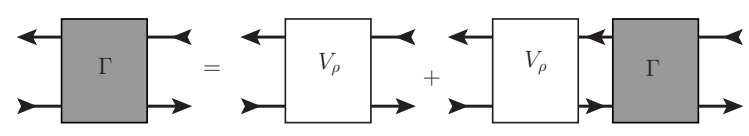

(c)
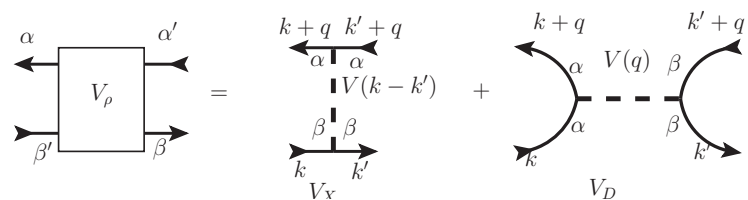

FIG. 2: (a) Diagrammatic representation of the dynamic charge susceptibility Eq. (20) in terms of the bare susceptibility and the interaction vertex $\Gamma\left(\mathbf{k}, \mathbf{k}^{\prime}, q\right)$. (b) Ladder diagrams corresponding to Eq. (17) for the interaction vertex. (c) The effective interaction in the charge channel, as in Eq. (11).

which measures the charge transfer between $\mathrm{O} p_{x}$ and $\mathrm{O} p_{y}$ orbitals. The corresponding nematic susceptibility is given by

$$
\begin{aligned}
\chi_{N}(\mathbf{q}) & =\frac{\partial O_{N}(\mathbf{q})}{\partial \phi(\mathbf{q})} \\
& =\chi_{x x}(\mathbf{q})-\chi_{x y}(\mathbf{q})+\chi_{y y}(\mathbf{q})-\chi_{y x}(\mathbf{q}) .
\end{aligned}
$$

$\phi(\mathbf{q})=\epsilon_{x}(\mathbf{q})-\epsilon_{y}(\mathbf{q})$ is a nematic perturbing potential, and the charge susceptibility matrix is

$$
\chi_{\alpha \beta}(\mathbf{q})=\frac{\delta n_{\alpha}(\mathbf{q})}{\delta \epsilon_{\beta}(\mathbf{q})},
$$

with $\delta n_{\alpha}(\mathbf{q})$ the change in the charge density $n_{\alpha}(\mathbf{q})=$ $\left\langle\hat{n}_{\alpha}(\mathbf{q})\right\rangle$ due to a weak perturbation $\delta \epsilon_{\beta}(\mathbf{q})$ of the orbital energies. For dynamical perturbations with frequency $\omega$, Eq. (10) generalizes to the dynamic susceptibility $\chi_{\alpha \beta}(\mathbf{q}, \omega)$. For the remainder of this section, we adopt the shorthand notation $q \equiv(\mathbf{q}, \omega)$.

The dynamic charge susceptibility is evaluated using the Kubo formula and an infinite summation of ladder and bubble diagrams, as shown in Fig. 2. For this purpose it is useful to define an effective interaction for the charge channel, $\underline{\underline{34}}$ as represented diagrammatically in Fig. 2(c),

$$
\begin{aligned}
V_{\rho, \alpha \alpha^{\prime} \beta \beta^{\prime}}\left(\mathbf{k}, \mathbf{k}^{\prime}, \mathbf{q}\right)= & -\delta_{\alpha^{\prime}, \alpha} \delta_{\beta^{\prime}, \beta} V_{\alpha \beta}\left(\mathbf{k}^{\prime}-\mathbf{k}\right) \\
& +\delta_{\alpha^{\prime}, \beta} \delta_{\beta^{\prime}, \alpha} 2 V_{\alpha \beta}(\mathbf{q}) .
\end{aligned}
$$

The first and the second term on the right describe the exchange and the direct interaction, respectively. Similarly, for the spin susceptibility the same set of diagrams is evaluated using the effective interaction in the spin channel, $\underline{34}$

$$
V_{\sigma, \alpha \alpha^{\prime} \beta \beta^{\prime}}\left(\mathbf{k}, \mathbf{k}^{\prime}, \mathbf{q}\right)=-\delta_{\alpha^{\prime}, \alpha} \delta_{\beta^{\prime}, \beta} V_{\alpha \beta}\left(\mathbf{k}^{\prime}-\mathbf{k}\right) .
$$

With Eq. (11), the interaction vertex $\Gamma_{\alpha \alpha^{\prime} \beta \beta^{\prime}}\left(\mathbf{k}, \mathbf{k}^{\prime}, q\right)$ in the charge channel is conveniently obtained in the compact form shown in Fig. 2(b). Once this equation is 
solved for $\Gamma_{\alpha \alpha^{\prime} \beta \beta^{\prime}}\left(\mathbf{k}, \mathbf{k}^{\prime}, q\right)$, the susceptibility is calculated from the diagrams in Fig. 2(a).

In order to solve the integral equation for the interaction vertex we project onto a set of basis functions in orbital and momentum space, $\stackrel{33}{=}$ which transforms the integral equation in Fig. 2(b) into a matrix equation:

$$
\begin{aligned}
V_{\alpha \beta}\left(\mathbf{k}^{\prime}-\mathbf{k}\right) & =\sum_{i, j} g_{\alpha \beta}^{i}(\mathbf{k}) \tilde{V}_{X}^{i j} g_{\alpha \beta}^{j}\left(\mathbf{k}^{\prime}\right), \\
V_{\alpha \beta}(\mathbf{q}) & =\sum_{i, j} g_{\alpha \alpha}^{i} \tilde{V}_{D}^{i j}(\mathbf{q}) g_{\beta \beta}^{j}, \\
\Gamma_{\alpha \alpha^{\prime} \beta \beta^{\prime}}\left(\mathbf{k}, \mathbf{k}^{\prime}, q\right) & =\sum_{i, j} g_{\alpha \beta^{\prime}}^{i}(\mathbf{k}) \tilde{\Gamma}^{i j}(q) g_{\alpha^{\prime} \beta}^{j}\left(\mathbf{k}^{\prime}\right) .
\end{aligned}
$$

The functions $g_{\alpha \beta}^{i}(\mathbf{k})$ form a 19-dimensional basis $(i \in$ $[1,19])$, and they are explicitly defined in Appendix $\mathbf{A} . \tilde{\mathbf{X}}$ denotes the matrix representation, with matrix elements $\tilde{X}^{i j}$, of a quantity $X$ with respect to the basis functions. In this notation $\tilde{\mathbf{V}}_{D}(\mathbf{q}), \tilde{\mathbf{V}}_{X}$, and $\tilde{\boldsymbol{\Gamma}}(q)$ are defined by Eqs. (13)-15). $\tilde{\mathbf{V}}_{D}(\mathbf{q})$ and $\tilde{\mathbf{V}}_{X}$ are also explicitly given in Appendix $\mathbf{A}$. We note that $\tilde{V}_{D}^{i j}(\mathbf{q})$ is nonzero only for $i, j=9,10,11$, for which $g_{\alpha \beta}^{i}(\mathbf{k})$ does not explicitly depend on $\mathbf{k}$.

Using Eqs. (13) and (14), we obtain

$$
\tilde{\mathbf{V}}_{\rho}(\mathbf{q})=2 \tilde{\mathbf{V}}_{D}(\mathbf{q})-\tilde{\mathbf{V}}_{X} .
$$

This equation, combined with Eq. (15), enables one to express the integral equation in Fig. 2(b) as a matrix equation, the inversion of which leads to

$$
\tilde{\boldsymbol{\Gamma}}(q)=\left[\mathbf{1}+\tilde{\mathbf{V}}_{\rho}(\mathbf{q}) \tilde{\boldsymbol{\chi}}_{0}(q)\right]^{-1} \tilde{\mathbf{V}}_{\rho}(\mathbf{q}) .
$$

$\tilde{\boldsymbol{\chi}}_{0}(q)$ is the projected bare susceptibility,

$$
\begin{aligned}
\tilde{\chi}_{0}^{i j}(q)=\frac{-1}{N} & \sum_{\mathbf{k}, \mu \nu} \sum_{\theta \theta^{\prime} \gamma \gamma^{\prime}} g_{\gamma^{\prime} \gamma}^{i}(\mathbf{k}) M_{\nu \mu \mathbf{k q}}^{\gamma^{\prime} \theta \theta^{\prime} \gamma} \\
& \times \frac{\left[f\left(E_{\mathbf{k}}^{\nu}\right)-f\left(E_{\mathbf{k}+\mathbf{q}}^{\mu}\right)\right]}{\omega+E_{\mathbf{k}}^{\nu}-E_{\mathbf{k}+\mathbf{q}}^{\mu}+\mathrm{i} \delta} g_{\theta \theta^{\prime}}^{j}(\mathbf{k}),
\end{aligned}
$$

with $f(E)$ the Fermi function and

$$
M_{\nu \mu \mathbf{k q}}^{\gamma^{\prime} \theta \theta^{\prime} \gamma}=S_{\gamma^{\prime} \nu}(\mathbf{k}) S_{\theta \nu}^{*}(\mathbf{k}) S_{\theta^{\prime} \mu}(\mathbf{k}+\mathbf{q}) S_{\gamma \mu}^{*}(\mathbf{k}+\mathbf{q}) .
$$

The charge susceptibility then follows as

$$
\chi_{\alpha \beta}(q)=\chi_{\alpha \beta}^{0}(q)-\sum_{i j} A_{\alpha \alpha}^{i}(q) \tilde{\boldsymbol{\Gamma}}^{i j}(q) A_{\beta \beta}^{j}(q),
$$

where

$$
\chi_{\alpha \beta}^{0}(q)=\frac{-1}{N} \sum_{\mathbf{k}, \mu \nu} M_{\nu \mu \mathbf{k q}}^{\beta \alpha \alpha \beta} \frac{\left[f\left(E_{\mathbf{k}}^{\nu}\right)-f\left(E_{\mathbf{k}+\mathbf{q}}^{\mu}\right)\right]}{\omega+E_{\mathbf{k}}^{\nu}-E_{\mathbf{k}+\mathbf{q}}^{\mu}+\mathrm{i} \delta}
$$

is the bare charge susceptibility, and

$A_{\gamma \gamma^{\prime}}^{j}(q)=\frac{1}{N} \sum_{\mathbf{k}, \mu \nu} \sum_{\theta \theta^{\prime}} M_{\nu \mu \mathbf{k q}}^{\gamma^{\prime} \theta \theta^{\prime} \gamma} \frac{f\left(E_{\mathbf{k}}^{\nu}\right)-f\left(E_{\mathbf{k}+\mathbf{q}}^{\mu}\right)}{\omega+E_{\mathbf{k}}^{\nu}-E_{\mathbf{k}+\mathbf{q}}^{\mu}+\mathrm{i} \delta} g_{\theta \theta^{\prime}}^{j}(\mathbf{k})$.

Equation (20), which corresponds to Fig. 2(a), is the final result for the charge susceptibility.

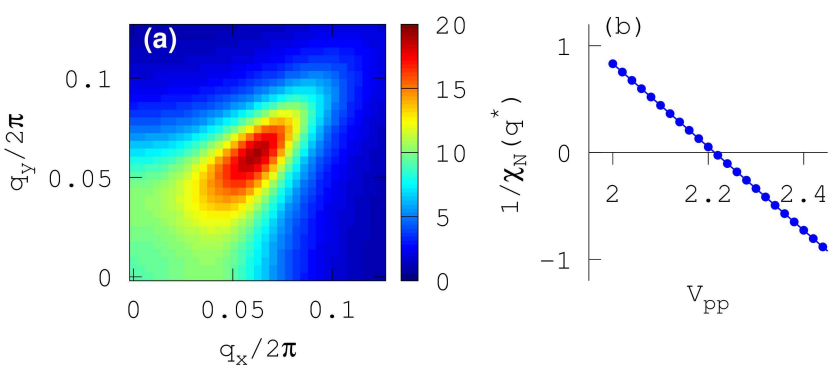

FIG. 3: (a) Nematic susceptibility $\chi_{N}(\mathbf{q})$ for $p=0.15$, $T=0.016, V_{p p}=2.2$, and $t_{p p}=0.5$. (b) $V_{p p}$ dependence of $1 / \chi_{N}\left(\mathbf{q}^{*}\right)$ showing the divergence of the susceptibility close to $V_{p p}=2.22$.

\section{RESULTS}

We identify charge instabilities from divergences of the charge susceptibility matrix. The focus is on nematic order that is marked by a divergence of $\chi_{N}(\mathbf{q})$ and driven by the repulsive interaction $V_{p p}$. We start with a general discussion of MNO and the structure of $\chi_{N}(\mathbf{q})$ and then present the results which show how the instability depends on $V_{p p}$, the hole density $p=5-n$, and the temperature T. $n$ is the number of electrons per unit cell, and $p$ is thus measured relative to half-filling of the $\mathrm{Cu} d$ orbital. We consider the two cases $t_{p p}=0$ and $t_{p p}=0.5$, which correspond to a small and a large Fermi surface curvature, respectively. The latter value is motivated by band-structure calculations and matches reasonably well the Fermi surface of cuprate materials. Subsequently we discuss the spectrum of the dynamical susceptibility $\chi_{N}(\mathbf{q}, \omega)$ in the isotropic phase near the nematic transition and the consequences of MNO for the reconstruction of the Fermi surface.

\section{A. Nematic Susceptibility}

As an example, we show in Fig. 3(a) the static nematic susceptibility $\chi_{N}(\mathbf{q})$ for a set of parameters near the MNO instability. For the selected parameters $\chi_{N}(\mathbf{q})$ is sharply peaked at the diagonal wavevector $\mathbf{q}^{*} / 2 \pi=$ $0.06(1,1)$. The inverse susceptibility at $\mathbf{q}^{*}$ is plotted in Fig. 3(b) as a function of $V_{p p}$; this figure shows that $\chi_{N}\left(\mathbf{q}^{*}\right)$ diverges at $V_{p p}=2.22$, which marks the boundary between an isotropic phase for $V_{p p}<2.22$ and MNO with a modulation wavevector $\mathbf{q}^{*}$. In fact, because $\chi_{N}(\mathbf{q})$ has the full point group symmetry of the lattice, it actually diverges simultaneously at four distinct $\mathbf{q}$-values related to $\mathbf{q}^{*}$ by $\pi / 2$ rotations. Our calculations therefore admit both unidirectional order (involving only $\mathbf{q}^{*}$ and $-\mathbf{q}^{*}$ ) and bidirectional checkerboard order (involving all four $\mathbf{q}$ vectors). Further extensions of the calculations are required to determine which phase is dominant, and in 
single-band models it has been shown that this depends on details of the interaction and Fermi surface $\stackrel{37}{ }$

Depending on model parameters, three kinds of nematic phases emerge from the calculations: a commensurate phase $\left(\mathbf{q}^{*}=\mathbf{0}\right)$, the diagonal phase with $\mathbf{q}^{*}=\left(q_{0}, q_{0}\right)$ as described above, and an axial phase for which the modulation wavevector is aligned with the crystalline axes, i.e. $\mathbf{q}^{*}=\left(q_{0}, 0\right)$ or $\mathbf{q}^{*}=\left(0, q_{0}\right)$. The charge modulations associated with both the diagonal and the axial phases are illustrated in Fig. 4. These figures are for model parameters near the MNO phase boundary, where the response to a weak perturbing potential is large. We show results for unidirectional and bidirectional nematic modulations: for the unidirectional case, the charge modulations are generated assuming a nematic perturbing potential of the form $\delta \epsilon_{d}=0$, $\delta \epsilon_{x}(\mathbf{r})=-\delta \epsilon_{y}(\mathbf{r})=\delta \epsilon \cos \left(\mathbf{q}^{*} \cdot \mathbf{r}\right)$, for which

$$
\delta n_{\alpha}(\mathbf{r})=\sum_{\beta} \chi_{\alpha \beta}\left(\mathbf{q}^{*}\right) \delta \epsilon_{\beta}(\mathbf{r}) .
$$

For the bidirectional case, a second perturbation, with $\mathbf{q}^{*}$ rotated by $\pi / 2$, is added to the right hand side of Eq. (23).

Figure 4 shows that in all cases, the charge transfer occurs almost entirely between the oxygen atoms; the $\mathrm{Cud}$ charge modulations are at least an order of magnitude smaller than the oxygen modulations and are too small to see in the figure. Furthermore, in each of the MNO patterns shown in Fig. 4 the charge transfer is predominantly within the unit cell, with the inter-unit cell charge transfers being orders of magnitude smaller. For this reason, it is natural to think of the broken symmetry phase as a modulated nematic rather than a CDW.

\section{B. Phase Boundaries}

We now examine how the phase boundary between isotropic and nematic phases depends on various model parameters. To begin with, we show in Figs. 5(a) and 6(a) the phase boundaries at low $T$ in the $p-V_{p p}$ plane for $t_{p p}=0$ and $t_{p p}=0.5$, respectively. In both figures, the system is isotropic for small $V_{p p}$, and the phase boundary marks where $\chi_{N}\left(\mathbf{q}^{*}\right)$ first diverges as $V_{p p}$ is increased. The value of $\mathbf{q}^{*}$ at which this happens depends on the hole density $p$, and is shown in Figs. 5(b) and 6)(b).

Figures 5(a) and 6(a) also include the curves along which $\chi_{N}(\mathbf{q}=\mathbf{0})$ diverges. We see that there is a wide range of $p$ over which $\chi_{N}\left(\mathbf{q}^{*}\right)$ diverges at a lower $V_{p p}$ than $\chi_{N}(\mathbf{0})$, signalling that MNO dominates over commensurate nematic order. There are also regions where only the $\mathbf{q}=\mathbf{0}$ instability is shown; in these regions this is the first instability which appears as $V_{p p}$ is increased.

The magnitude and the orientation of $\mathbf{q}^{*}$ depend on the filling relative to the "van Hove filling" $p_{\mathrm{vH}}$, which is defined as the hole density $p$ at which the van Hove singularities at $(\pi, 0)$ and $(0, \pi)$ cross the Fermi energy.
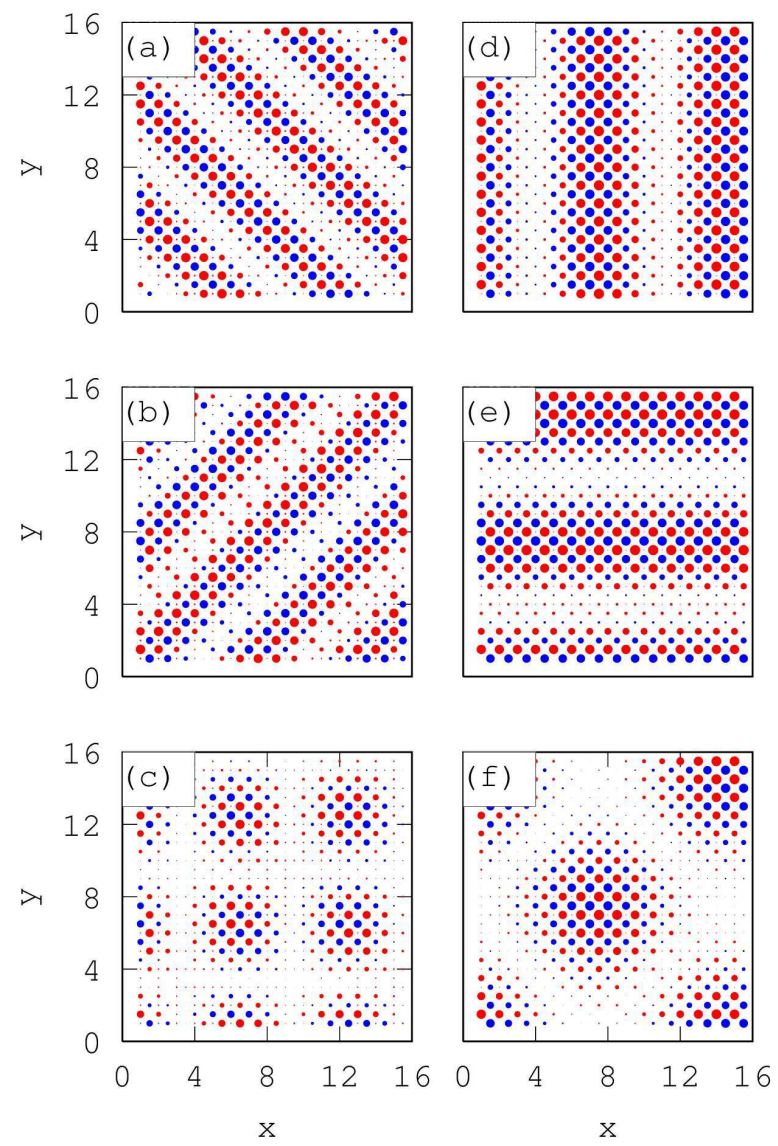

FIG. 4: Charge density modulations for (a)-(c) diagonal and (d)-(f) axial modulated nematic order, at hole densities $p=0.14$ and $p=0.20$, respectively, for $t_{p p}=0.5$. The corresponding $q^{*}$ values are given in Fig. 6] (b). Circle diameter indicates the magnitude of the charge modulation, with red (blue) corresponding to a negative (positive) modulation. While all orbitals are shown, modulations are only large enough to be seen on the oxygen $p_{x}$ and $p_{y}$ orbitals. The bidirectional patterns in (c) are obtained by adding the patterns in (a) and (b); those in (f) are obtained by adding (d) and (e).

$p_{\mathrm{vH}}$ marks the boundary between hole-like $\left(p<p_{\mathrm{vH}}\right)$ and electron-like $\left(p>p_{\mathrm{vH}}\right)$ Fermi surfaces. The value of $p_{\mathrm{vH}}$ depends on the Fermi surface curvature: $p_{\mathrm{vH}}=0$ for $t_{p p}=0$ and $p_{\mathrm{vH}}=0.177$ for $t_{p p}=0.5$. For reference, cuprate superconductors have hole-like Fermi surfaces in the doping range where charge ordered phases are observed.

In both Figs. 5] and [6, the nematic instability is to a commensurate phase at large $p$. At lower fillings, but still above $p_{\mathrm{vH}}$, the leading instability is incommensurate, with an axial modulation wavevector that decreases as $p$ is reduced: when $t_{p p}=0, \mathbf{q}^{*}$ vanishes continuously as $p \rightarrow p_{v H}$, at which point the charge order is commensurate. For $t_{p p}=0.5$, there is a discontinuous transition to 

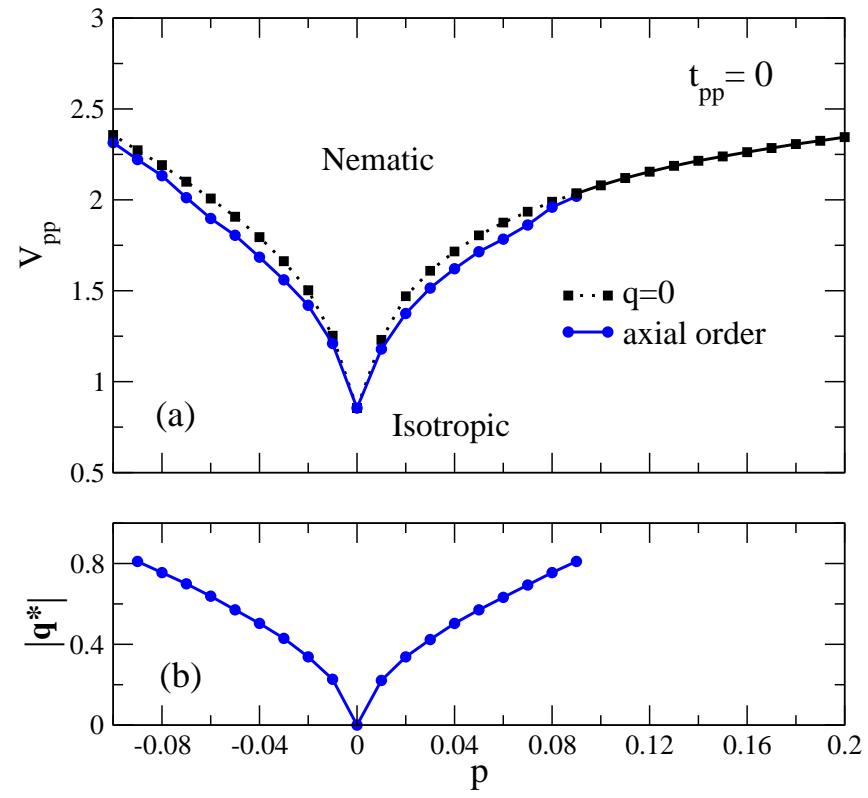

FIG. 5: (a) Charge instabilities for the commensurate $(\mathbf{q}=$ 0) and axial modulated nematic phases for $t_{p p}=0$ at $T=$ 0.0005 . The system is isotropic for small $V_{p p}$. Solid curves indicate the leading divergence of $\chi_{N}(\mathbf{q})$ as $V_{p p}$ is increased; dashed curves indicate subleading instabilities. The phase transition is thus to an axial nematic phase for $p \leq 0.09$, and to a commensurate nematic phase for $p>0.09$. The modulation wavevector for the axial nematic phase is shown in (b), and it vanishes at the van Hove filling $p_{\mathrm{vH}}=0.0$. The model parameters are $t_{p d}=1, \epsilon_{d}=0, \Delta_{C T}=\epsilon_{d}-\epsilon_{p}=2.5$, $V_{p d}=1, U_{d}=9$, and $U_{p}=3$.

the commensurate phase at $p=0.19>p_{\mathrm{vH}}$. Below the van Hove filling, $\left|\mathbf{q}^{*}\right|$ grows as $p$ is reduced. In this range of doping the modulation wavevector is axial for $t_{p p}=0$ (Fig. 5) and diagonal for $t_{p p}=0.5$ (Fig. 6). For the relative hole filling $p=p_{v H}-0.05$, the crossover between diagonal and axial order occurs for $t_{p p} \approx 0.12$. As we discuss further in Sec. IVA the overall doping dependence is similar to that found by Holder and Metzner ${ }^{39}$

In Fig. 7 we map the nematic instability in the $p$ - $T$ plane for fixed $V_{p p}$. Results for $t_{p p}=0$ and $t_{p p}=0.5$ are shown in Figs. Z(a) and (b), respectively. In both cases, the system is isotropic at high $T$, and the leading instability upon cooling is indicated by a solid line; dashed lines indicate subleading instabilities.

Figure 7(a) is dominated by a transition to commensurate nematic order extending over a broad doping range that includes both hole-like $(p<0)$ and electron-like $(p>0)$ Fermi surfaces. The system exhibits re-entrance at both the lower and upper ends of the doping range where nematic order is encountered: as $T$ is lowered, the system passes through an isotropic-to-nematic transition followed by a nematic-to-isotropic transition. The shape of the commensurate phase boundary is essentially the same as that found in a previous mean-field study $\stackrel{35}{\underline{3}}$ The new result in Fig. (7)(a) is the existence of a phase bound-
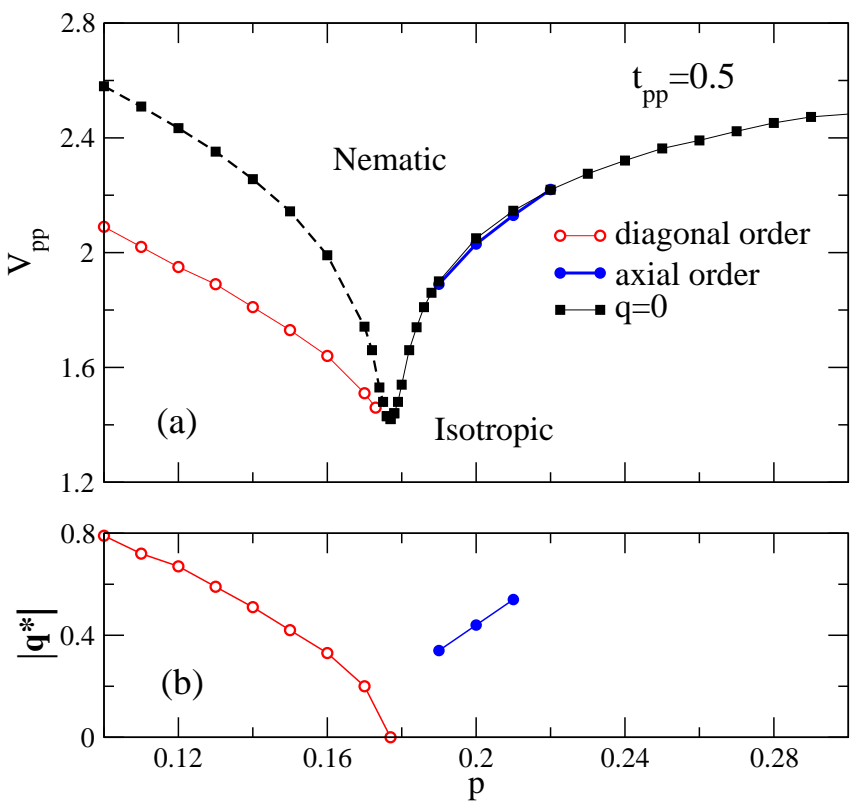

FIG. 6: As in Fig. 5 but for $t_{p p}=0.5$. Here, the leading instability is to a diagonal nematic phase for $p<p_{\mathrm{vH}}$, where $p_{\mathrm{vH}}=0.177$ for this value of $t_{p p}$.

ary spanning $-0.07<p<-0.062$ between isotropic and axial MNO phases at low $T$. This figure shows that the axial MNO phase found at low $T$ (Fig. 5) is fragile for $t_{p p}=0$ and subdominant to the commensurate phase at higher $T$.

While the MNO phase boundary represents only a small fraction of Fig. 7(a), it is much more important when we adopt the more realistic value of $t_{p p}=0.5$ in Fig. 7(b). In particular, the leading instability is to a diagonal MNO phase for a wide doping range of hole-like Fermi surfaces $\left(p<p_{\mathrm{vH}}\right)$. This doping range is similar to the range over which charge modulations are observed experimentally. However, there are two significant discrepancies with the experiments: the values of $\mathbf{q}^{*}$ obtained here are a factor of 2 to 3 smaller than those observed experimentally, and the orientation of $\mathbf{q}^{*}$ is diagonal, while experiments observe axial order. The first discrepancy might be resolved by using a band structure tailored specifically to ortho-II YBCO $\stackrel{49}{\sharp}$ this likely requires going beyond the three-band model. These issues will be discussed in more detail in Sec. IVB.

Finally, we note that the nematic instability is actually subleading for our model parameters; the leading instability is to a spin-density wave (SDW) state. A calculation of the spin susceptibility, performed in the same manner as for the charge susceptibility, but using the effective interaction in the spin channel Eq. (12) in place of Eq. (11), shows that the SDW involves primarily the $\mathrm{Cu} d$ orbitals and is driven by the large on-site repulsion $U_{d}$ on the copper atoms. For $p=0.14, t_{p p}=0.5$, and $V_{p p}=2.2$, the onset temperature for the SDW is higher than the nematic transition temperature provided 

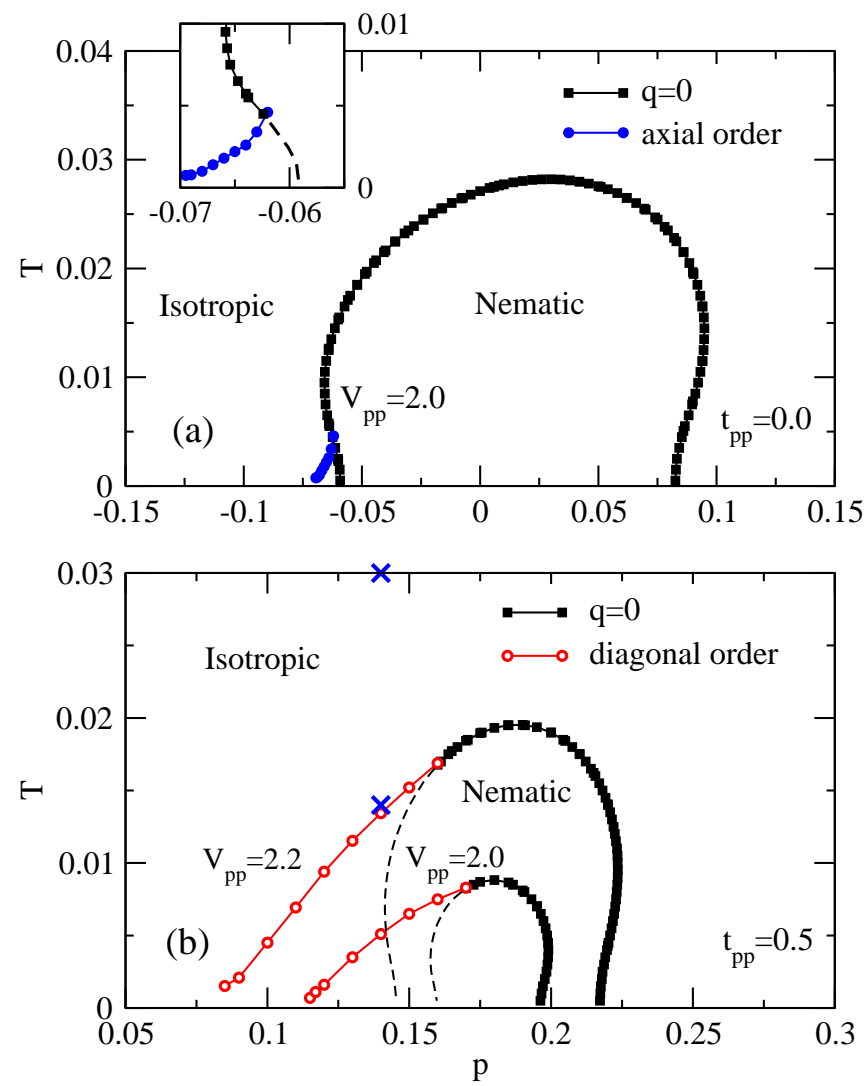

FIG. 7: Phase diagram in the $p$ - $T$ plane for (a) $t_{p p}=0$ and (b) $t_{p p}=0.5$. Solid curves indicate the leading divergence of $\chi_{N}(\mathbf{q})$ upon cooling; dashed lines indicate subleading instabilities. The inset in (a) shows a zoom into the left corner of the nematic region where axial order is dominant. The crosses in (b) indicate the points in the phase diagram at which the dynamic susceptibility is shown in Fig. 8. Model parameters are as in Fig. 5

$U_{d}>1.08$ while for smaller $U_{d}$, the nematic instability occurs at higher $T$. There are two reasons why this SDW phase may not matter for nematic order. First, strong correlation effects, neglected here, will renormalize the interaction vertex, and thereby lead to a lower magnetic transition temperature than predicted by weak-coupling theory. Second, the onset of SDW order does not preclude nematic order becase the SDW and MNO involve different oribitals. This raises the intriguing possibility that these phases might coexist, with little or no competition, in some regions of the phase diagram.

\section{Dynamical Susceptibility}

Figure 8 shows the dynamical susceptibility at the modulation wavevector $\mathbf{q}^{*}$ in the isotropic phase. Results are shown for the same parameters as in Fig. 7(b), with $p=0.14, V_{p p}=2.2$, and for $T=0.014$ and $T=0.030$. These filling and temperature values are indicated by crosses in Fig. 7(b). The lower temperature, $T=0.014$,
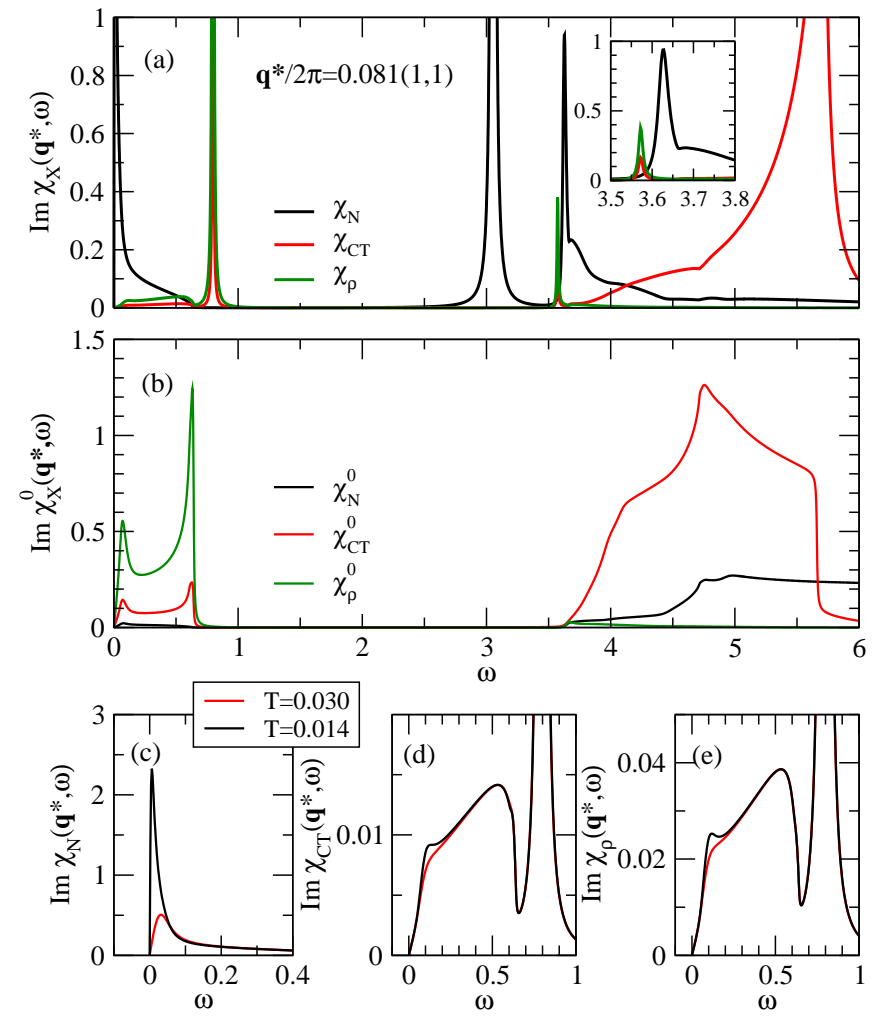

FIG. 8: Imaginary part of the frequency dependent susceptibility at $\mathbf{q}^{*}$. Results are for $t_{p p}=0.5$, hole density $p=0.14$, and $V_{p p}=2.2$. (a) Plots of the nematic, the charge-transfer, and the charge susceptibility slightly above the nematic transition $(T=0.014)$. The inset shows an expanded view of the frequency range $3.5<\omega<3.8$. For comparison, the bare susceptibilities are shown in (b). The temperature dependence of the low-frequency spectrum is separately displayed for the nematic (c), the charge-transfer (d) and the charge susceptibility (e). Panel (c) shows the emergence of a sharp low-frequency peak in the nematic susceptibility near the nematic transition. For reference, the points on the phase diagram at which the susceptibilities are evaluated are marked with crosses in Fig. 7(b).

is close to the nematic transition while the higher temperature, $T=0.030$, is approximately twice the transition temperature.

Besides the nematic susceptibility $\chi_{N}(\mathbf{q}, \omega)$, the total charge susceptibility

$$
\chi_{\rho}(\mathbf{q}, \omega)=\frac{\partial\left(n_{d}+n_{x}+n_{y}\right)}{\partial\left(\epsilon_{d}+\epsilon_{x}+\epsilon_{y}\right)}=\sum_{\alpha, \beta} \chi_{\alpha \beta}(\mathbf{q}, \omega)
$$

and the charge-transfer susceptibility ${ }^{34}$

$$
\begin{aligned}
\chi_{\mathrm{CT}}(\mathbf{q}, \omega) & =\frac{\partial\left(n_{d}-n_{x}-n_{y}\right)}{\partial\left(\epsilon_{d}-\epsilon_{x}-\epsilon_{y}\right)} \\
& =\chi_{\rho}(\mathbf{q}, \omega)-2 \sum_{\alpha=x, y}\left[\chi_{d \alpha}(\mathbf{q}, \omega)+\chi_{\alpha d}(\mathbf{q}, \omega)\right] .
\end{aligned}
$$

are included. The frequency dependence of these three susceptibilities are shown in Fig. 8) (a) in comparison to 
the bare versions of these susceptibilities in Fig. 8 (b). The bare spectra consist of a low-energy intraband continuum due to particle-hole excitations with momentum $\mathbf{q}^{*}$, and a high-energy interband continuum. The width of the low-energy continuum is q-dependent, and vanishes as $q \rightarrow 0$; the interband particle-hole continuum is instead only weakly q-dependent.

The particle-hole continuum is renormalized by the interactions. In particular, the low-frequency charge susceptibility $\chi_{\rho}\left(\mathbf{q}^{*}, \omega\right)$ is reduced by an order of magnitude relative to $\chi_{\rho}^{0}\left(\mathbf{q}^{*}, \omega\right)$. This originates from the large value of the on-site Coulomb interaction, $U_{d}=9$, which suppresses charge fluctuations on the $\mathrm{Cu} d$ orbitals. The susceptibilities in Fig. 8(a) have a number of additional resonances. Just above the low-frequency continuum, at $\omega=0.8$, both $\chi_{\mathrm{CT}}\left(\mathbf{q}^{*}, \omega\right)$ and $\chi_{\rho}\left(\mathbf{q}^{*}, \omega\right)$ exhibit a sharp resonance, which was identified before as a zero-sound mode $\stackrel{50}{=}$ It is this mode, rather than the charge-transfer excitation, which becomes soft at the charge-transfer instability. However, this mode is not relevant for the nematic transition as it remains at finite frequency across the transition.

The second pronounced resonance, at $\omega=5.58$, is the charge-transfer excitation. This mode corresponds to a transfer of charge between $\mathrm{Cu} d$ and $\mathrm{O} p$ orbitals without any change in the total intra-unit cell charge density; consequently, the peak appears in $\chi_{\mathrm{CT}}\left(\mathbf{q}^{*}, \omega\right)$ but not in $\chi_{\rho}\left(\mathbf{q}^{*}, \omega\right)$. A third excitonic resonance appears in both $\chi_{\mathrm{CT}}\left(\mathbf{q}^{*}, \omega\right)$ and $\chi_{\rho}\left(\mathbf{q}^{*}, \omega\right)$ at $\omega=3.57$, just below the high-frequency interband continuum. As shown in the inset, this excitonic peak is distinct from a nearby nematic resonance at $\omega=3.63$. All three modes (zero sound, excitonic, and charge transfer) have $A_{1 g}$ symmetry as $q \rightarrow 0$. In contrast, resonances in the nematic susceptibility have $B_{1 g}$ symmetry. This is the same symmetry as for the $d$-wave Pomeranchuk instability $: 51,52$

Three resonances are visible in the nematic susceptibility. There are two excitonic resonances at $\omega=3.63$ and $\omega=3.05$ and a low-frequency peak that becomes soft at the nematic transition. All three excitations involve only $\mathrm{O} p_{x}$ and $\mathrm{O} p_{y}$ orbitals, such that there is no peak in $\chi_{\alpha \beta}\left(\mathbf{q}^{*}, \omega\right)$, if either $\alpha=d$ or $\beta=d$.

As shown in Fig. 8(c)-(e) the low-frequency spectra of both $\chi_{\rho}\left(\mathbf{q}^{*}, \omega\right)$ and $\chi_{\mathrm{CT}}\left(\mathbf{q}^{*}, \omega\right)$ are only weakly $T$ dependent. The low-frequency nematic mode, on the other hand, depends strongly on $T$. In particular, it is damped by the particle-hole continuum away from the nematic transition, but sharpens significantly as $T$ is lowered, and the excitation frequency shifts towards zero. The approach to the nematic transition is therefore accompanied by the softening of a mode that emerges from the particle-hole continuum.

\section{Fermi Surface in the MNO Phase}

To understand the effects of modulated nematic order on the single-particle spectrum, we recalculate the
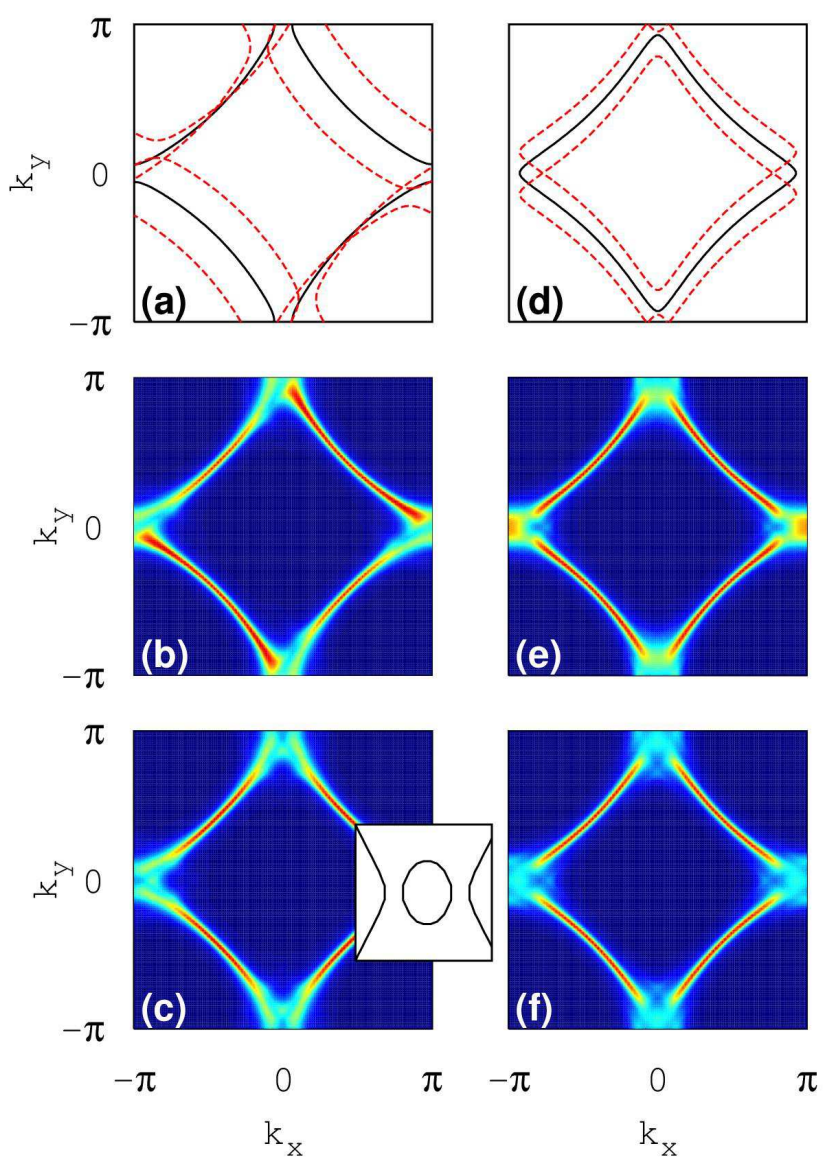

FIG. 9: Fermi surface reconstruction for (a)-(c) diagonal ( $p=$ $0.14)$ and (d)-(f) axial $(p=0.20)$ modulation vectors $\mathbf{q}^{*}$ for $t_{p p}=0.5$. Solid black lines in (a) and (d) show Fermi surface contours, and dotted red lines show the same contours shifted by $\pm \mathbf{q}^{*}$. Intensity plots in (b),(c),(e),(f) show the spectral function, $A\left(\mathbf{k}, \varepsilon_{F}\right)$. Results are for (b),(e) unidirectional and (c),(f) bidirectional modulations. In (c), the inset shows a zoom of the reconstructed Fermi surface, centered at $(\pi, 0)$. In all panels, $\mathbf{q}^{*}$ is taken from Fig. 6 for the corresponding filling. A Lorentzian broadening of $\delta=0.05$ was used to calculate the spectral functions.

band structure of the three-band model in the presence of nematic order $\delta \epsilon_{x}(\mathbf{r})=-\delta \epsilon_{y}(\mathbf{r})=\delta \epsilon \cos \left(\mathbf{q}^{*} \cdot \mathbf{r}\right)$, in the same fashion used to generate Fig. 4 . For the unidirectional modulations, we start from an approximate perturbed Hamiltonian of the form

$$
\mathbf{H}(\mathbf{k})=\left[\begin{array}{ccc}
\mathbf{H}_{0}(\mathbf{k}) & \mathbf{H}_{\mathbf{q}^{*}} & \mathbf{H}_{-\mathbf{q}^{*}} \\
\mathbf{H}_{\mathbf{q}^{*}} & \mathbf{H}_{0}\left(\mathbf{k}+\mathbf{q}^{*}\right) & 0 \\
\mathbf{H}_{-\mathbf{q}^{*}} & 0 & \mathbf{H}_{0}\left(\mathbf{k}-\mathbf{q}^{*}\right)
\end{array}\right],
$$

where $\mathbf{H}_{0}(\mathbf{k})$ is the same $3 \times 3$ matrix as in Eq. (2). $\mathbf{H}_{ \pm \mathbf{q}^{*}}=(\delta \epsilon / 2) \operatorname{diag}(0,1,-1)$ is the matrix representation of the perturbing potential, which scatters quasiparticles by $\pm \mathbf{q}^{*} . \mathbf{H}(\mathbf{k})$ is a $9 \times 9$ matrix that is diagonalized 
numerically for each $\mathbf{k}$. In principle, incommensurate nematic order also mixes in states with momenta $\mathbf{k} \pm 2 \mathbf{q}^{*}$, $\mathbf{k} \pm 3 \mathbf{q}^{*}$, etc.. However, we find that these states rapidly diminish in importance and that the spectral function can be understood in terms of the leading order terms alone.

The Fermi surfaces are obtained from the eigenvalues of $\mathbf{H}(\mathbf{k})$, while the spectral function is taken from the trace over $\mathrm{Cu} d, \mathrm{O} p_{x}$, and $\mathrm{O} p_{y}$ orbitals, namely

$$
A(\mathbf{k}, \omega)=-\frac{\operatorname{Im}}{\pi} \sum_{i=1}^{3}[(\omega+\mathrm{i} \delta) \mathbf{1}-\mathbf{H}(\mathbf{k})]_{i i}^{-1}
$$

where $[\ldots]_{i i}^{-1}$ is the $i$ th diagonal element of the matrix inverse. The Fermi surfaces and the spectral functions are plotted in Fig. 9 in the first Brillouin zone. The same two cases are considered here as in Fig. 4. $p=0.14<$ $p_{\mathrm{vH}}$, for which $\mathbf{q}^{*}$ is diagonal, and $p=0.20>p_{\mathrm{vH}}$, for which $\mathbf{q}^{*}$ is axial; the values for $\mathbf{q}^{*}$ are taken from Fig. 6.

In Figs. 9(a) and (d), we show contours of the bare Fermi surface, along with Fermi-surface replicas shifted by $\pm \mathbf{q}^{*}$. In Fig. 9(a), the bare and shifted Fermi surface segments coincide near $(\pi, 0)$ and $(0, \pi)$ (the antinodes in the language of $d$-wave superconductivity), which suggests that the diagonal nematic modulation is driven by nesting of short Fermi surface segments in the antinodal regions. Nesting features are not obvious in Fig. 9(d), but there is a short segment of the Fermi surface near $(0, \pi)$ that coincides with (and is tangential to) one of the shifted Fermi surfaces.

In Figs. 9(b) and 9(e) we map $A(\mathbf{k}, \omega)$ at the respective Fermi energies $\varepsilon_{F}$ for the same modulation vectors as in (a) and (b). The spectral functions both exhibit a depletion of spectral weight near the antinodes and the residual spectral weight lies mostly along Fermi surface arcs, in broad agreement with angle-resolved photoemission spectroscopy (ARPES) experiments 53 ARPES has shown that the pseudogap in underdoped cuprates is generically characterized by a gap on the antinodal portion of the Fermi surface.

One widely studied scenario for the gap invokes the proximity of the underdoped cuprates to an antiferromagnetic insulating phase, and assumes either static or dynamical SDW correlations with a modulation wavevector near $(\pi, \pi)$. These kinds of scenarios generically lead to a spectral function with four hole-like Fermi surface pockets 54 centered near $( \pm \pi / 2, \pm \pi / 2)$, but such pockets have yet to be observed experimentally. In contrast, scenarios in which $\mathbf{q}^{*}$ nests antinodal Fermi surface sections do not generate nodal pockets; consistent with this the spectral functions in Fig. 9(b) and (e) have no backfolding around $( \pm \pi / 2, \pm \pi / 2)$.

It is obvious from Figs. 9(b) and 9(e) that unidirectional MNO leads to an orthorhombic distortion of the Fermi surface. Four-fold rotational symmetry is restored when the MNO is bidirectional, as illustrated by Figs. 9(c) and 9(f). The spectral functions for bidirectional MNO are qualitatively similar to the unidirectional

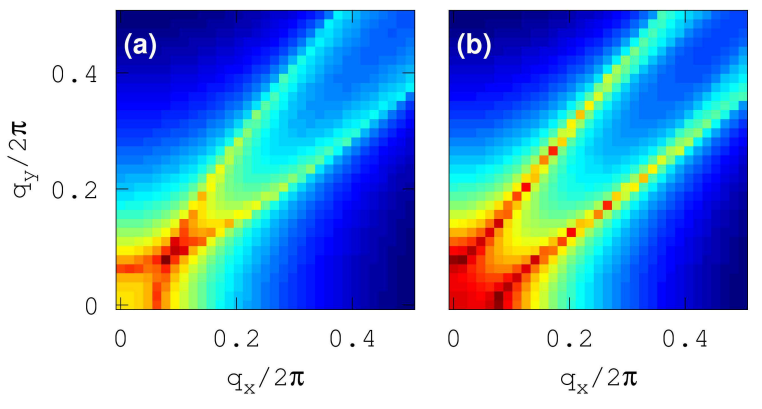

FIG. 10: Bare nematic susceptibility $\chi_{1 \mathrm{~B}}^{0}(\mathbf{q})$ for the conduction band of the three-band model. The susceptibility is calculated from Eq. (28), with $\epsilon_{\mathbf{k}}$ set equal to the largest eigenvalue $E_{\mathbf{k}}^{3}$ of the Hamiltonian $\mathbf{H}_{0}(\mathbf{k})$. Results are shown for hole dopings (a) $p=0.14<p_{\mathrm{vH}}$ and (b) $p=0.20>p_{\mathrm{vH}}$, with $t_{p p}=0.5$.

cases, with one notable exception: the reconstructed Fermi surface in Fig. 9(c) has small electron-like pockets that close around $(\pi, 0)$ and $(0, \pi)$. These pockets have low spectral weight, and therefore do not show up strongly in the spectral function. One of the pockets is shown in the inset to Fig. 9(c), where a portion of the reconstructed Fermi surface is plotted. We discuss these pockets further in Sec. IVB.

\section{DISCUSSION}

\section{A. Comparison to One-Band Models}

In this section we address the doping dependence of the modulation wavevector $\mathbf{q}^{*}$. We have noted that for $t_{p p}=0.5$, axial MNO is preferred for $p>p_{\mathrm{vH}}$ and diagonal MNO is preferred for $p<p_{\mathrm{vH}}$ as was also obtained by Holder and Metzner ${ }^{39}$ In their one-band model the nematic instabilities involve a spontaneous symmetry breaking between the $x$ - and $y$-axis bond order parameters $\left\langle c_{i+x \sigma}^{\dagger} c_{i \sigma}\right\rangle$ and $\left\langle c_{i+y \sigma}^{\dagger} c_{i \sigma}\right\rangle$, while in the three-band model, the instability involves a transfer of charge between $\mathrm{O} p_{x}$ and $\mathrm{O} p_{y}$ orbitals.

As emphasized in Ref. 39, the nematic instability in the one-band model is understood from the peak structure of the $d$-wave polarization function

$$
\chi_{1 \mathrm{~B}}^{0}(\mathbf{q})=-\frac{1}{N} \sum_{\mathbf{k}} \frac{f\left(\epsilon_{\mathbf{k}-\mathbf{q} / 2}\right)-f\left(\epsilon_{\mathbf{k}+\mathbf{q} / 2}\right)}{\epsilon_{\mathbf{k}-\mathbf{q} / 2}-\epsilon_{\mathbf{k}+\mathbf{q} / 2}} d_{\mathbf{k}}^{2}
$$

where $\epsilon_{\mathbf{k}}$ is the band dispersion and $d_{\mathbf{k}}=\cos \left(k_{x}\right)-$ $\cos \left(k_{y}\right)$ is a $d$-wave form factor. $\chi_{1 B}^{0}(\mathbf{q})$ is illustrated in Fig. 10 for electron densities below and above the van Hove filling. For this figure, we have set $\epsilon_{\mathbf{k}}=E_{\mathbf{k}}^{3}$, where $E_{\mathbf{k}}^{3}$ is the conduction band dispersion for the three-band model, obtained by diagonalizing $\mathbf{H}_{0}(\mathbf{k})$.

The $\mathbf{q}$-space arcs along which $\chi_{1 \mathrm{~B}}^{0}(\mathbf{q})$ is peaked in Fig. 10 correspond to the Fermi surface nesting condi- 
tion $E_{\mathbf{k}}^{3}=E_{\mathbf{k}+\mathbf{q}}^{3}=\varepsilon_{F}$, with $\varepsilon_{F}$ the Fermi energy. For hole-like Fermi surfaces, these arcs cross [Fig. 10(a)] so that a single peak on the Brillouin zone diagonal emerges at the crossing point $\mathbf{q}^{*}$. For electron-like Fermi surfaces instead, the arcs do not cross [Fig. 10(b)] and two maxima lie on the $x$ - and the $y$-axis, respectively. While this simple analysis explains the doping dependence of $\mathbf{q}^{*}$ in the one-band model, its applicability to the three-band model is not at all obvious.

The bare susceptibility in the three-band model is a $3 \times 3$ matrix,

$$
\begin{aligned}
\chi_{\theta \gamma}^{0}(\mathbf{q})= & -\frac{1}{N} \sum_{\mathbf{k}} \sum_{\mu, \nu=1}^{3}\left|S_{\gamma \nu}\left(\mathbf{k}-\frac{\mathbf{q}}{2}\right) S_{\theta \mu}\left(\mathbf{k}+\frac{\mathbf{q}}{2}\right)\right|^{2} \\
& \times \frac{f\left(E_{\mathbf{k}-\mathbf{q} / 2}^{\nu}\right)-f\left(E_{\mathbf{k}+\mathbf{q} / 2}^{\mu}\right)}{E_{\mathbf{k}-\mathbf{q} / 2}^{\nu}-E_{\mathbf{k}+\mathbf{q} / 2}^{\mu}}
\end{aligned}
$$

where $\mathbf{S}(\mathbf{k})$ is the unitary matrix defined by Eq. (3) that diagonalizes $\mathbf{H}_{0}(\mathbf{k})$. Empirically, the most important contributions to the nematic susceptibility [Eq. (9)] are from $\chi_{x x}^{0}(\mathbf{q})$ and $\chi_{y y}^{0}(\mathbf{q})$, which are at least an order of magnitude larger than $\chi_{x y}^{0}(\mathbf{q})$ and $\chi_{y x}^{0}(\mathbf{q})$. These two dominant contributions are illustrated in Figs. 11(a)-(d) for fillings below and above the van Hove filling.

The most important contribution to $\chi_{\theta \gamma}^{0}(\mathbf{q})$ in Eq. (29) comes from intraband transitions in the conduction band $(\mu=\nu=3)$. Focusing on this contribution alone, the main distinction between Eq. (29) and Eq. (28) is the weighting factor, which in the one-band case is $d_{\mathbf{k}}^{2}$ and in the three-band case consists of a product of matrix elements of $\mathbf{S}(\mathbf{k})$. As is apparent from the plots of $\chi_{x x}^{0}(\mathbf{q})$ and $\chi_{y y}^{0}(\mathbf{q})$ in Fig. 11, these matrix elements break the fourfold rotational symmetry of the underlying band structure. Rotational symmetry is restored, however, for the bare nematic susceptibility, which is approximately determined by $\chi_{N}^{0}(\mathbf{q}) \approx \chi_{x x}^{0}(\mathbf{q})+\chi_{y y}^{0}(\mathbf{q})$.

Similarly, the full nematic susceptibility has a fourfold rotational symmetry, although the structure is somewhat different from that of $\chi_{N}^{0}(\mathbf{q})$. To show this, following Bang et al. $\stackrel{50}{=}$ we evaluate Eq. (20) for a reduced basis set comprising the most important basis functions, $g_{\alpha \beta}^{9}, g_{\alpha \beta}^{10}$, and $g_{\alpha \beta}^{11}$. To obtain a qualitative picture of the transition, we keep only the contribution of $V_{p p}$, which drives the charge instability, and set $U_{d}=V_{p d}=U_{p}=0$. The interaction in the charge channel is thus reduced to

$$
\tilde{\mathbf{V}}_{\rho}=2 \tilde{\mathbf{V}}_{\mathbf{D}}-\tilde{\mathbf{V}}_{\mathbf{X}}=\left[\begin{array}{ccc}
0 & 0 & 0 \\
0 & 0 & \bar{V}_{p p} \\
0 & \bar{V}_{p p} & 0
\end{array}\right],
$$

where $\bar{V}_{p p}=8 V_{p p} \cos \left(q_{x} / 2\right) \cos \left(q_{y} / 2\right)$. The transition to a charge ordered state occurs when the smallest eigenvalue of the matrix $\mathbf{D}=\mathbf{1}+\tilde{\mathbf{V}}_{\rho} \tilde{\boldsymbol{\chi}}_{0}$, the inverse of which enters into Eq. (17), vanishes. For the reduced basis and the simplified interaction, this matrix is

$$
\mathbf{D}=\left[\begin{array}{ccc}
1 & 0 & 0 \\
\bar{V}_{p p} \chi_{d y}^{0} & 1 & \bar{V}_{p p} \chi_{y y}^{0} \\
\bar{V}_{p p} \chi_{d x}^{0} & \bar{V}_{p p} \chi_{x x}^{0} & 1
\end{array}\right]
$$
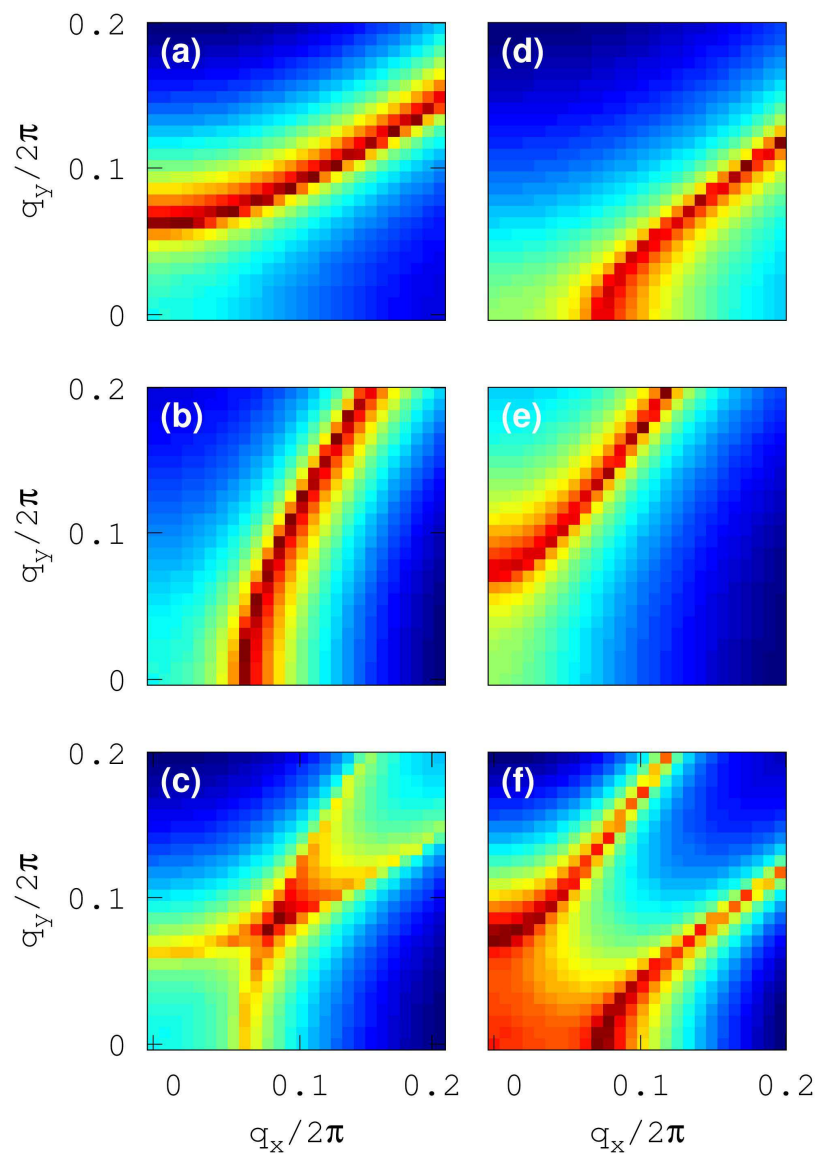

FIG. 11: Plots of (a),(d) $\chi_{x x}^{0}(\mathbf{q}),(\mathrm{b}),(\mathrm{e}) \chi_{y y}^{0}(\mathbf{q})$, and (c),(f) $\bar{\chi}^{0}(\mathbf{q})[\mathrm{Eq}$. (32)] for $T=0.0005$ and fillings (a)-(c) $p=0.14$ and (d)-(f) $p=0.20$.

It is straightforward to show that the smallest eigenvalue of $\mathbf{D}$ is $1-\bar{V}_{p p} \bar{\chi}^{0}(\mathbf{q})$, where

$$
\bar{\chi}^{0}(\mathbf{q})=\sqrt{\chi_{x x}^{0}(\mathbf{q}) \chi_{y y}^{0}(\mathbf{q})}
$$

is the geometric average of $\chi_{x x}^{0}(\mathbf{q})$ and $\chi_{y y}^{0}(\mathbf{q})$. The quantity $\bar{\chi}^{0}(\mathbf{q})$ can be interpreted as the bare susceptibility for the charge mode associated with the leading nematic instability. As one can see from a comparison of Figs. 10(a),(b) and Figs. 11(e),(f), $\bar{\chi}^{0}(\mathbf{q})$ and $\chi_{1 \mathrm{~B}}^{0}(\mathbf{q})$ are qualitatively similar. In particular, $\bar{\chi}^{0}(\mathbf{q})$ has the same arc structure as in the one-band model: $\mathbf{q}^{*}$ lies along the Brillouin zone diagonal when the arcs cross (hole-like Fermi surface) and it lies on the Brillouin zone axes when the arcs do not cross (electron-like Fermi surface). The connection between the direction of $\mathbf{q}^{*}$ and the Fermi surface topology thus appears to be a robust feature. 


\section{B. Comparison to Experiments}

Our calculations lead to an incommensurate modulation with a doping dependent $\mathbf{q}^{*}$. Experimentally, there has been some debate about the value and the doping dependence of the modulation wavevector. NMR studies of ortho-II YBCO $\left(\mathrm{YBa}_{2} \mathrm{Cu}_{3} \mathrm{O}_{6.54}\right)$ found that the planar $\mathrm{Cu}$ NMR line is split into two distinct peaks corresponding to $\mathrm{Cu}$ sites below empty $(\mathrm{E})$ and filled $(\mathrm{F})$ $\mathrm{CuO}$ chains (in ortho-II YBCO, every second chain has no oxygen), and that the NMR line corresponding to $\mathrm{Cu}-$ E sites develops a double-peak at the onset of charge order 11 This was interpreted in terms of commensurate period- 4 order, with $q^{*}=0.25$ in units of $2 \pi / a_{0}$, where $a_{0}$ is the lattice constant. Subsequent x-ray scattering experiments 15,16 found incommensurate modulations with $q^{*} \approx 0.32$. This appears at first sight to be inconsistent with the NMR experiments because an incommensurate modulation should produce a continuous, rather than bimodal, distribution of $\mathrm{Cud}$ charge densities. However, it was recently pointed out ${ }^{55}$ that the distribution for an incommensurate charge modulation has a double-peak structure provided the modulations are unidirectional. To illustrate this, we plot in Fig. 12 the normalized histograms of both the $\mathrm{Cu} d$ and the $\mathrm{O} p$ chargedensity modulations shown in Figs. 4(a) and 4(c). For the unidirectional MNO, Fig. 12(a), the distribution has the double-peak structure described above. The width of the distribution is larger for the $\mathrm{O} p$ orbitals than for the $\mathrm{Cud}$ orbitals because nematic order involves primarily the oxygen sites. The double-peak structure is not present when the MNO is bidirectional, Fig.12(b), so consistency with NMR requires that ortho-II charge modulations be unidirectional. Recent x-ray experiments by Blackburn et al. did indeed observe that charge modulations in ortho-II YBCO are predominantly along the $b$-axis 17

The same series of $\mathrm{x}$-ray experiments finds modulation wavevectors along both the $a$ and $b$ directions at higher dopings. An interesting question, yet to be addressed, is whether NMR is consistent with these experiments. In particular, NMR experiments should be able to determine, based on whether the lineshape resembles those in Figs. 12(a) or 12(b), whether the two $q^{*}$ values correspond to spatially separated domains of unidirectional order or to bidirectional order.

The doping dependence of $q^{*}$ also needs to be resolved experimentally. The MNO described by our calculations originates from a Fermi surface instability and $q^{*}$ therefore has a strong doping dependence: for a hole-like Fermi surface, the modulation wavevector decreases as the hole density increases and vanishes at $p_{\mathrm{vH}}$. Ghiringhelli et $a l .15$ did not find any doping dependence to the modulation wavevector, but Blackburn et al ${ }^{17}$ found a $\sim 10 \%$ decrease in $q^{*}$ as the hole doping was increased from 0.104 to 0.132 (a 30\% change). Although the variation of $q^{*}$ with doping is slower than predicted by our calculations, the general trend is qualitatively consistent. The doping dependence of checkerboard modulations has also been
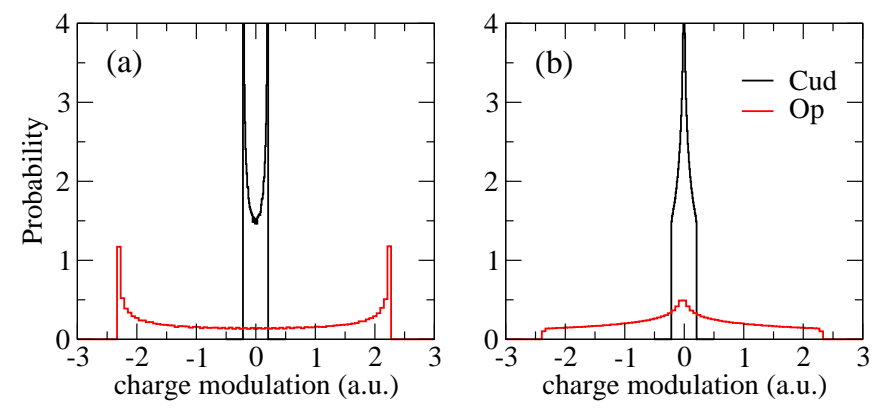

FIG. 12: Normalized histograms of the charge modulations in Fig. 4 for $p=0.14$ (diagonal MNO) with (a) unidirectional and (b) bidirectional modulations. Histograms are shown for both $\mathrm{Cud}$ and $\mathrm{O} p$ orbitals. The distributions for axial MNO are similar.

explored by STM in other materials: the modulation wavevector was found to be weakly doping dependent in $\mathrm{Bi}_{2} \mathrm{Sr}_{2} \mathrm{CaCu}_{2} \mathrm{O}_{8+\delta}(\mathrm{Bi} 2212)^{56}$ and in $\mathrm{Ca}_{2-\mathrm{x}} \mathrm{Na}_{\mathrm{x}} \mathrm{CuO}_{2} \mathrm{Cl}_{2}$ (Ref. 57), but is much more strongly doping dependent in $\mathrm{Bi}_{2-\mathrm{y}} \mathrm{Pb}_{\mathrm{y}} \mathrm{Sr}_{2-\mathrm{z}} \mathrm{La}_{\mathrm{z}} \mathrm{CuO}_{6+\mathrm{x}}(\mathrm{Bi} 2201)^{27}$. In $\mathrm{Bi} 2201$, the modulation wavevector decreased from $2 \pi / 4.5 a_{0}$ to $2 \pi / 6.2 a_{0}$ as the doping was changed from underdoped $\left(T_{c}=25 \mathrm{~K}\right)$ to optimal $\left(T_{c}=35 \mathrm{~K}\right)$. These $q^{*}$ values are much smaller than what is found in YBCO, and are quantitatively close to what we have found, though the ordering is rotated by $45^{\circ}$.

As we have mentioned above, the two main discrepancies between our calculations and the experiments on YBCO are that for hole-like Fermi surfaces the calculated orientation of $q^{*}$ is diagonal rather than axial, and that the magnitude of $q^{*}$ is too small by a factor of 2 or 3. The magnitude of $q^{*}$ in our calculations is set by the Fermi surface structure, and it is entirely possible that the discrepancy may be reconciled by tailoring the model specifically to ortho-II YBCO. Such a model could include, for example, additional Fermi surface sheets due to $\mathrm{CuO}$ chains, or to the fact that each unit cell contains two $\mathrm{CuO}_{2}$ planes. With the exception of ortho-II YBCO, the $\mathrm{CuO}$ chains seem unlikely to resolve the discrepancies because charge order is observed along both $a$ and $b$ axes in detwinned crystals, where the chains lie along the $b$ direction. The bilayer structure, however, may be important. Ghiringhelli et al. noted that the observed $q^{*}$ is generally too large to be due to nesting of a single $\mathrm{CuO}_{2}$ Fermi surface, and proposed instead that $q^{*}$ connects segments of the bonding Fermi surface of the $\mathrm{CuO}_{2}$ bilayer. To explore this, we have repeated our calculations for a single $\mathrm{CuO}_{2}$ bilayer, and have found that (i) it is possible for the susceptibility to be larger on the bonding sheet than on the antibonding sheet but that (ii) we always obtain diagonal MNO for hole-like Fermi surfaces. As discussed in the previous section, the trend for holelike Fermi surfaces to have a diagonal modulation vector is a remarkably robust feature of our calculation.

Finally, we return to the discussion of the Fermi surface in the modulated nematic phase. As we noted 
in Sec. IIID the spectral intensity is suppressed in the antinodal region, and is therefore broadly consistent with photoemission experiments on underdoped cuprates. We also noted that for hole-like Fermi surfaces (corresponding to underdoped cuprates) the reconstructed Fermi surface has small electron-like pockets near $(\pi, 0)$ and $(0, \pi)$. This is particularly interesting because magneto-oscillation experiments, $\stackrel{6,7,58-63}{\underline{6}}$ along with Hall measurements, $\frac{8}{\underline{y}}$ on YBCO have demonstrated the existence of small electron-like Fermi surface pockets occupying $\sim 2 \%$ of the Brillouin zone area. We make a few observations about a possible correspondence between the experiments and our calculations. First, while electron pockets should be observable in ARPES experiments, such experiments are difficult to perform on YBCO. Second, a large number of ARPES experiments has been done on Bi-based cuprates, and these have not seen electron pockets. However, Bi-cuprates are highly inhomogeneous and this can mask spectral signatures of non-superconducting phases $\underline{54}$ Furthermore, magneto-oscillation experiments are hampered in the Bicuprates by high levels of disorder, and have not provided independent confirmation of the Fermi surface structure. It is thus possible that charge modulations are accompanied by well-defined antinodal electron pockets in YBCO but not in Bi-cuprates. Third, the structure and the existence of the predicted pockets in our calculations depend on the size and the orientation of $\mathbf{q}^{*}$. At present it is unclear whether a model that correctly predicts the orientation of $\mathbf{q}^{*}$ will also generate an antinodal electron pocket.

\section{CONCLUSIONS}

We have calculated, within a weak-coupling diagrammatic perturbation theory, the charge susceptibility matrix $\chi_{\alpha \beta}(\mathbf{q}, \omega)$, from which we have identified several nematic charge instabilities in the three-band Emery model. Taking model parameters appropriate for cuprate superconductors, we find that there is a broad doping range over which the model is unstable towards a modulated nematic phase, characterized by a charge transfer between oxygen $p_{x}$ and $p_{y}$ orbitals. Such a phase has many features consistent with experiments. However, in the relevant doping range, the orientation of the modulation wavevector is rotated with respect to that measured in x-ray scattering experiments. At this point, it is not clear whether this discrepancy is the result of an oversimplification of the cuprate band structure by the threeband model, or whether it is the result of still unresolved physics that is missing from the model.

\section{Acknowledgments}

We thank B. M. Andersen and M.-H. Julien for helpful conversations. W.A.A. acknowledges support by the Natural Sciences and Engineering Research Council (NSERC) of Canada. This work was made possible by the facilities of the Shared Hierarchical Academic Research Computing Network and Compute/Calcul Canada. S.B. acknowledges a German Academic Exchange Service (DAAD) Research Grant PKZ1072498 received in the year 2011. A.P.K. acknowledges support by the DFG through TRR 80.

\section{Appendix A: Function Basis}

The momentum dependent interaction is separable in the following basis:

$$
\begin{aligned}
g_{\alpha \beta}^{1} & =g_{\beta \alpha}^{12}=\delta_{\alpha d} \delta_{\beta x} \cos \left(k_{x} / 2\right), \\
g_{\alpha \beta}^{2} & =g_{\beta \alpha}^{13}=\delta_{\alpha d} \delta_{\beta x} \sin \left(k_{x} / 2\right), \\
g_{\alpha \beta}^{3} & =g_{\beta \alpha}^{14}=\delta_{\alpha d} \delta_{\beta y} \cos \left(k_{y} / 2\right), \\
g_{\alpha \beta}^{4} & =g_{\beta \alpha}^{15}=\delta_{\alpha d} \delta_{\beta y} \sin \left(k_{y} / 2\right), \\
g_{\alpha \beta}^{5} & =g_{\beta \alpha}^{16}=\delta_{\alpha x} \delta_{\beta y} \cos \left(k_{x} / 2\right) \cos \left(k_{y} / 2\right), \\
g_{\alpha \beta}^{6} & =g_{\beta \alpha}^{17}=\delta_{\alpha x} \delta_{\beta y} \cos \left(k_{x} / 2\right) \sin \left(k_{y} / 2\right), \\
g_{\alpha \beta}^{7} & =g_{\beta \alpha}^{18}=\delta_{\alpha x} \delta_{\beta y} \sin \left(k_{x} / 2\right) \cos \left(k_{y} / 2\right), \\
g_{\alpha \beta}^{8} & =g_{\beta \alpha}^{19}=\delta_{\alpha x} \delta_{\beta y} \sin \left(k_{x} / 2\right) \sin \left(k_{y} / 2\right), \\
g_{\alpha \beta}^{9} & =\delta_{\alpha d} \delta_{\beta d}, \\
g_{\alpha \beta}^{10} & =\delta_{\alpha x} \delta_{\beta x}, \\
g_{\alpha \beta}^{11} & =\delta_{\alpha y} \delta_{\beta y} .
\end{aligned}
$$

For example, the $p$ - $d$ density-density interaction $V_{x d}(\mathbf{q})=2 V_{p d} \cos \left(q_{x} / 2\right)$ is

$$
\begin{aligned}
V_{x d}\left(\mathbf{k}-\mathbf{k}^{\prime}\right)= & 2 V_{p d} \cos \left(k_{x} / 2-k_{x}^{\prime} / 2\right) \\
= & 2 V_{p d}\left\{\cos \left(k_{x} / 2\right) \cos \left(k_{x}^{\prime} / 2\right)\right. \\
& \left.+\sin \left(k_{x} / 2\right) \sin \left(k_{x}^{\prime} / 2\right)\right\} \\
= & \sum_{i=12}^{13} g_{x d}^{i}(\mathbf{k}) \tilde{V}_{X}^{i i} g_{x d}^{i}\left(\mathbf{k}^{\prime}\right)
\end{aligned}
$$

where

$$
\tilde{V}_{X}^{i j}=\left\{\begin{array}{cc}
2 V_{p d} \delta_{i j}, & i \in\{1 \ldots 4,12 \ldots 15\} \\
2 V_{p p} \delta_{i j}, & i \in\{5 \ldots 8,16 \ldots 19\} \\
U_{d} \delta_{i j}, & i=9 \\
U_{p} \delta_{i j}, & i=10,11
\end{array}\right.
$$

is the exchange interaction matrix in this basis. The direct interaction $\tilde{V}_{D}^{i j}(\mathbf{q})$ is zero everywhere except for $i, j \in\{9,10,11\}$. In this $3 \times 3$ block

$$
\tilde{V}_{D}(\mathbf{q})=\left(\begin{array}{ccc}
U_{d} & 2 V_{p d} \mathrm{c}_{x}(\mathbf{q}) & 2 V_{p d} \mathrm{c}_{y}(\mathbf{q}) \\
2 V_{p d} \mathrm{c}_{x}(\mathbf{q}) & U_{p} & 4 V_{p p} \mathrm{c}_{x}(\mathbf{q}) \mathrm{c}_{y}(\mathbf{q}) \\
2 V_{p d} \mathrm{c}_{y}(\mathbf{q}) & 4 V_{p p} \mathrm{c}_{x}(\mathbf{q}) \mathrm{c}_{y}(\mathbf{q}) & U_{p}
\end{array}\right)
$$

where $\mathrm{c}_{x}(\mathbf{q})=\cos \left(q_{x} / 2\right)$ and $\mathrm{c}_{y}(\mathbf{q})=\cos \left(q_{y} / 2\right)$. 
* Electronic address: billatkinson@trentu.ca

1 J. M. Tranquada, B. J. Sternlieb, J. D. Axe, Y. Nakamura, and S. Uchida, Nature 375, 561 (1995).

2 P. Abbamonte, A. Rusydi, S. Smadici, G. D. Gu, G. A. Sawatzky, and D. L. Feng, Nat. Phys. 1, 155 (2005).

3 M. Vojta, Advances in Physics 58, 699 (2009).

4 J. M. Tranquada, G. D. Gu, M. Hücker, Q. Jie, H.-J. Kang, R. Klingeler, Q. Li, N. Tristan, J. S. Wen, G. Y. Xu, Z. J. $\mathrm{Xu}$, J. Zhou, and M. v. Zimmermann, Phys. Rev. B 78, 174529 (2008)

${ }^{5}$ M. Hücker, M. v. Zimmermann, Z. J. Xu, J. S. Wen, G. D. Gu, and J. M. Tranquada, Phys. Rev. B 87, 014501 (2013).

6 N. Doiron-Leyraud, C. Proust, D. LeBoeuf, J. Levallois, J.-B. Bonnemaison, R. Liang, D. A. Bonn, W. N. Hardy, and L. Taillefer, Nature 447, 565 (2007).

7 S. E. Sebastian, N. Harrison, and G. Lonzarich, Rep. Prog. Phys. 75, 102501 (2012).

8 D. LeBoeuf, N. Doiron-Leyraud, J. Levallois, R. Daou, J. B. Bonnemaison, N. E. Hussey, L. Balicas, B. J. Ramshaw, R. Liang, D. A. Bonn, W. N. Hardy, S. Adachi, C. Proust, and L. Taillefer, Nature 450, 533 (2007).

9 S. Chakravarty, Rep. Prog. Phys. 74, 022501 (2011).

10 R. Daou, J. Chang, D. LeBoeuf, O. Cyr-Choinière, F. Laliberté, N. Doiron-Leyraud, B. J. Ramshaw, R. Liang, D. A. Bonn, W. N. Hardy, and L. Taillefer, Nature 463, 519 (2010).

11 T. Wu, H. Mayaffre, S. Krämer, M. Horvatić, C. Berthier, W. N. Hardy, R. Liang, D. A. Bonn, and M.-H. Julien, Nature 477, 191 (2011).

12 V. Hinkov, P. Bourges, S. Pailhès, Y. Sidis, A. Ivanov, C. D. Frost, T. G. Perring, C. T. Lin, D. P. Chen, and B. Keimer, Nat. Phys. 3, 780 (2007).

13 V. Hinkov, D. Haug, B. Fauqué, P. Bourges, Y. Sidis, A. Ivanov, C. Bernhard, C. T. Lin, and B. Keimer, Science 319, 597 (2008).

14 D. Haug, V. Hinkov, Y. Sidis, P. Bourges, N. B. Christensen, A. Ivanov, T. Keller, C. T. Lin, and B. Keimer, New J. Phys. 12, 105006 (2010).

15 G. Ghiringhelli, M. Le Tacon, M. Minola, S. BlancoCanosa, C. Mazzoli, N. B. Brookes, G. M. De Luca, A. Frano, D. G. Hawthorn, F. He, T. Loew, M. M. Sala, D. C. Peets, M. Salluzzo, E. Schierle, R. Sutarto, G. A. Sawatzky, E. Weschke, B. Keimer, and L. Braicovich, Science 337, 821 (2012).

16 J. Chang, E. Blackburn, A. T. Holmes, N. B. Christensen, J. Larsen, J. Mesot, R. Liang, D. A. Bonn, W. N. Hardy, A. Watenphul, M. v. Zimmermann, E. M. Forgan, and S. M. Hayden, Nat. Phys. 8, 871 (2012).

17 E. Blackburn, J. Chang, M. Hücker, A. T. Holmes, N. B. Christensen, R. Liang, D. A. Bonn, W. N. Hardy, U. Rütt, O. Gutowski, M. v. Zimmermann, E. M. Forgan, and S. M. Hayden, Phys. Rev. Lett. 110, 137004 (2013).

18 D. LeBoeuf, S. Krämer, W. N. Hardy, R. Liang, D. A. Bonn, and C. Proust, Nat. Phys. 9, 79 (2013).

19 S. Blanco-Canosa, A. Frano, T. Loew, Y. Lu, J. Porras, G. Ghiringhelli, M. Minola, C. Mazzoli, L. Braicovich, E. Schierle, E. Weschke, M. Le Tacon, and B. Keimer, Phys. Rev. Lett. 110, 187001 (2013).

20 T. Wu, H. Mayaffre, S. Krämer, M. Horvatić, C. Berthier, P. L. Kuhns, A. P. Reyes, R. Liang, W. N. Hardy, D. A. Bonn, and M.-H. Julien, Nat. Commun. 4 (2013).
21 T. Kondo, R. Khasanov, T. Takeuchi, J. Schmalian, and A. Kaminski, Nature (London) 457, 296 (2009).

22 M. Hashimoto, R.-H. He, K. Tanaka, J.-P. Testaud, W. Meevasana, R. G. Moore, D. Lu, H. Yao, Y. Yoshida, H. Eisaki, T. P. Devereaux, Z. Hussain, and Z.-X. Shen, Nat. Phys. 6, 414 (2010).

23 R.-H. He, M. Hashimoto, H. Karapetyan, J. D. Koralek, J. P. Hinton, J. P. Testaud, V. Nathan, Y. Yoshida, H. Yao, K. Tanaka, W. Meevasana, R. G. Moore, D. H. Lu, S.K. Mo, M. Ishikado, H. Eisaki, Z. Hussain, T. P. Devereaux, S. A. Kivelson, J. Orenstein, A. Kapitulnik, and Z.-X. Shen, Science 331, 1579 (2011).

24 S.-I. Ideta, T. Yoshida, A. Fujimori, H. Anzai, T. Fujita, A. Ino, M. Arita, H. Namatame, M. Taniguchi, Z.-X. Shen, K. Takashima, K. Kojima, and S.-I. Uchida, Phys. Rev. B 85, 104515 (2012).

25 J. E. Hoffman, E. W. Hudson, K. M. Lang, V. Madhavan, H. Eisaki, S. Uchida, and J. C. Davis, Nature 295, 466 (2002).

26 C. Howald, H. Eisaki, N. Kaneko, M. Greven, and A. Kapitulnik, Phys. Rev. B 67, 014533 (2003).

27 W. D. Wise, M. C. Boyer, K. Chatterjee, T. Kondo, T. Takeuchi, H. Ikuta, Y. Wang, and E. W. Hudson, Nat. Phys. 4, 696 (2008).

28 C. V. Parker, P. Aynajian, E. H. da Silva Neto, A. Pushp, S. Ono, J. Wen, Z. Xu, G. Gu, and A. Yazdani, Nature 468, 677 (2010).

29 M. J. Lawler, K. Fujita, J. Lee, A. R. Schmidt, Y. Kohsaka, C. K. Kim, H. Eisaki, S. Uchida, J. C. Davis, J. P. Sethna, and E.-A. Kim, Nature 466, 347 (2010).

30 A. Mesaros, K. Fujita, H. Eisaki, S. Uchida, J. C. Davis, S. Sachdev, J. Zaanen, M. J. Lawler, and E.-A. Kim, Science 333, 426 (2011).

31 E. H. da Silva Neto, C. V. Parker, P. Aynajian, A. Pushp, A. Yazdani, J. Wen, Z. Xu, and G. Gu, Phys. Rev. B 85, 104521 (2012).

${ }^{32}$ V. J. Emery, Phys. Rev. Lett. 58, 2794 (1987).

33 P. B. Littlewood, C. M. Varma, S. Schmitt-Rink, and E. Abrahams, Phys. Rev. B 39, 12371 (1989).

34 P. B. Littlewood, Phys. Rev. B 42, 10075 (1990).

35 M. H. Fischer and E.-A. Kim, Phys. Rev. B 84, 144502 (2011).

36 M. A. Metlitski and S. Sachdev, New J. Phys. 12, 105007 (2010).

37 A. Melikyan and M. R. Norman (2011), http://arxiv.org/abs/1102.5443.

38 C. Husemann and W. Metzner, Phys. Rev. B 86, 085113 (2012).

39 T. Holder and W. Metzner, Phys. Rev. B 85, 165130 (2012).

40 K. B. Efetov, H. Meier, and C. Pépin, Nature Physics 9, 442 (2013).

41 S. Sachdev and R. La Placa, Phys. Rev. Lett. 111, 027202 (2013).

42 H. Meier, M. Einenkel, C. Pépin, and K. B. Efetov, Phys. Rev. B 88, 020506 (2013).

43 R. T. Scalettar, D. J. Scalapino, R. L. Sugar, and S. R. White, Phys. Rev. B 44, 770 (1991).

44 S. A. Kivelson, E. Fradkin, and T. H. Geballe, Phys. Rev. B 69, 144505 (2004).

${ }^{45}$ K. Sun, B. M. Fregoso, M. J. Lawler, and E. Fradkin, Phys. 
Rev. B 78, 085124 (2008).

46 O. Andersen, A. Liechtenstein, O. Jepsen, and F. Paulsen, J. Phys. Chem. Solids 56, 1573 (1995).

47 E. Arrigoni, M. Aichhorn, M. Daghofer, and W. Hanke, New J. Phys. 11, 055066 (2009).

48 M. S. Hybertsen, M. Schlüter, and N. E. Christensen, Phys. Rev. B 39, 9028 (1989).

49 A. Carrington and E. A. Yelland, Phys. Rev. B 76, 140508 (2007).

50 Y. Bang, G. Kotliar, R. Raimondi, C. Castellani, and M. Grilli, Phys. Rev. B 47, 3323 (1993).

51 H. Yamase, Phys. Rev. Lett. 93, 266404 (2004).

${ }^{52}$ H. Yamase, V. Oganesyan, and W. Metzner, Phys. Rev. B 72, 035114 (2005).

53 A. Damascelli, Z. Hussain, and Z.-X. Shen, Rev. Mod. Phys. 75, 473 (2003).

${ }^{54}$ W. A. Atkinson, J. D. Bazak, and B. M. Andersen, Phys. Rev. Lett. 109, 267004 (2012).

55 S. Hayden, APS March Meeting, URL http://meetings .aps.org/link/BAPS . 2013.MAR.B2.2

56 Y. Kohsaka, C. Taylor, P. Wahl, A. Schmidt, J. Lee, K. Fujita, J. W. Alldredge, K. McElroy, J. Lee, H. Eisaki, S. Uchida, D.-H. Lee, and J. C. Davis, Nature 454, 1072 (2008).
57 T. Hanaguri, C. Lupien, Y. Kohsaka, D. Lee, M. Azuma, M. Takano, H. Takagi, and J. Davis, Nature 430, 1001 (2004).

58 S. C. Riggs, O. Vafek, J. B. Kemper, J. B. Betts, A. Migliori, F. F. Balakirev, W. N. Hardy, R. Liang, D. A. Bonn, and G. S. Boebinger, Nat. Phys. 7, 332 (2011).

59 J. Singleton, C. de la Cruz, R. D. McDonald, S. Li, M. Altarawneh, P. Goddard, I. Franke, D. Rickel, C. H. Mielke, X. Yao, and P. Dai, Phys. Rev. Lett. 104, 086403 (2010).

60 A. F. Bangura, J. D. Fletcher, A. Carrington, J. Levallois, M. Nardone, B. Vignolle, P. J. Heard, N. Doiron-Leyraud, D. LeBoeuf, L. Taillefer, S. Adachi, C. Proust, and N. E. Hussey, Phys. Rev. Lett. 100, 047004 (2008).

61 E. Yelland, J. Singleton, C. Mielke, N. Harrison, F. Balakirev, B. Dabrowski, and J. Cooper, Phys. Rev. Lett. 100, 047003 (2008).

62 S. E. Sebastian, N. Harrison, E. Palm, T. P. Murphy, C. H. Mielke, R. Liang, D. A. Bonn, W. N. Hardy, and G. G. Lonzarich, Nature 454, 200 (2008).

63 S. Sebastian, N. Harrison, R. Liang, D. Bonn, W. Hardy, C. Mielke, and G. Lonzarich, Phys. Rev. Lett. 108, 196403 (2012). 